This is a postprint version of the following published document:

Ibort, A.; Lledó, F.; Pérez-Pardo, J. M. (2015). "On Self-Adjoint Extensions and Symmetries in Quantum Mechanics". Annales Henri Poincaré, v. 16, Issue 10, pp. 2367-2397. DOI: $10.1007 / \mathrm{s} 00023-014-0379-4$

(C) Springer 2015 


\title{
On Self-Adjoint Extensions and Symmetries in Quantum Mechanics
}

\author{
Alberto Ibort, Fernando Lledó and Juan Manuel Pérez-Pardo
}

\begin{abstract}
Given a unitary representation of a Lie group $G$ on a Hilbert space $\mathcal{H}$, we develop the theory of $G$-invariant self-adjoint extensions of symmetric operators using both von Neumann's theorem and the theory of quadratic forms. We also analyze the relation between the reduction theory of the unitary representation and the reduction of the $G$-invariant unbounded operator. We also prove a $G$-invariant version of the rep-resentation theorem for closed and semi-bounded quadratic forms. The previous results are applied to the study of $G$-invariant self-adjoint exten-sions of the Laplace-Beltrami operator on a smooth Riemannian manifold with boundary on which the group $G$ acts. These extensions are labeled by admissible unitaries $U$ acting on the $L^{2}$-space at the boundary and having spectral gap at -1 . It is shown that if the unitary representation $V$ of the symmetry group $G$ is traceable, then the self-adjoint extension of the Laplace-Beltrami operator determined by $U$ is $G$-invariant if $U$ and $V$ commute at the boundary. Various significant examples are discussed at the end.
\end{abstract}

\section{Introduction}

Symmetries of quantum mechanical systems are described by a group of transformations that preserves its essential structures. They play a fundamental role in studying the properties of the quantum system and reveal fundamental aspects of the theory which are not present neither in the dynamics involved nor in the forces. Space or time symmetries, internal symmetries, the study

A. Ibort and J. M. Pérez-Pardo are partly supported by the project MTM2010-21186-C0202 of the spanish Ministerio de Ciencia e Innovación and QUITEMAD programme P2009 ESP-1594. F. Lledó was partially supported by projects DGI MICIIN MTM2012-36372-C0301 and Severo Ochoa SEV-2011-0087 of the spanish Ministry of Economy and Competition. J. M. Pérez-Pardo was also partially supported in 2011 and 2012 by mobility grants of the "Universidad Carlos III de Madrid" . 
of invariant states or spontaneously broken symmetries are standard ingredients in the description of quantum theories. In many cases, quantum numbers or superselection rules are labels characterizing representations of symmetry groups. The publication of the seminal books of Weyl, Wigner and van der Waerden (cf. $[37,38,40])$ in the late twenties also indicates that quantum mechanics was using group theoretical methods almost from its birth. We refer, e.g., to [25, Chapter 12] or [31] for a more thorough introduction to various symmetry notions in quantum mechanics.

It was shown by Wigner that any symmetry transformation of a quantum system preserving the transition probabilities between two states must be implemented by a semi-unitary (i.e., by a unitary or an anti-unitary) operator (see, e.g., [39, Introduction] or [32, Chapters 2]). The action of a symmetry group $G$ on a system is given in terms of a semi-unitary projective representation of $G$ on the physical Hilbert space, that can be described in terms of semiunitary representations of $U(1)$-central extensions of the group or by means of an appropriate representation group (see, for instance, $[6,11,12]$ ). Since the main examples of symmetries considered in this article will be implemented in terms of unitary operators we will restrict here to this case. Moreover, antiunitary representation appear rarely in applications (typically implementing time reversal) and restrict to discrete groups. The situation with an antiunitary representation of a discrete symmetry group can also be easily incorporated in our approach.

In order to motivate how the symmetry can be implemented at the level of unbounded operators, consider a self-adjoint Hamiltonian $T$ on the Hilbert space $\mathcal{H}$ and let $U(t):=e^{i t T}$ be the strongly continuous one-parameter group implementing the unitary evolution of the quantum system. Then, if $G$ is a quantum symmetry represented by the unitary representation $V: G \rightarrow \mathcal{U}(\mathcal{H})$ it is natural to require that $V$ and $U$ commute, i.e.,

$$
U(t) V(g)=V(g) U(t), \quad t \in \mathbb{R}, g \in G .
$$

At the level of self-adjoint generators and, recalling that the domain of $T$ is given by

$$
\mathcal{D}(T):=\left\{\psi \in \mathcal{H} \mid \lim _{t \rightarrow 0} \frac{(U(t)-\mathbb{I}) \psi}{t} \text { exists }\right\},
$$

we have that (1.1) implies

$$
V(g) \mathcal{D}(T) \subset \mathcal{D}(T) \quad \text { and } \quad V(g) T \psi=T V(g) \psi, \quad \psi \in \mathcal{D}(T) .
$$

Of course, the requirement that the unitary representation $V$ of the symmetry group $G$ commutes with the dynamics of the system as in Eq. (1.1) is restrictive. For example, if $V$ is a strongly continuous representation of a Lie group, then (1.1) implies the existence of conserved quantities that do not depend explicitly on time. Nevertheless, the previous comments justify that in the context of a single unbounded symmetric operator $T$ (not necessarily a Hamiltonian) it is reasonable to define $G$-invariance of $T$ as in Eq. (1.2) (see Sect. 3 for details). 
In the study of quantum systems it is standard that some heuristic arguments suggest an expression for an observable which is only symmetric on an initial dense domain but not self-adjoint. The description of such systems is not complete until a self-adjoint extension of the operator has been determined, e.g., a self-adjoint Hamiltonian operator T. Only in this case, a unitary evolution of the system is given. This is due to the one-to-one correspondence between densely defined self-adjoint operators and strongly continuous oneparameter groups of unitary operators $U_{t}=\exp i t T$ provided by Stone's theorem. The specification of a self-adjoint extension is typically done by choosing suitable boundary conditions and this corresponds to a global understanding of the system (see, e.g., [19,20] and references therein). Accordingly, the specification of the self-adjoint extension is not just a mathematical technicality, but a crucial step in the description of the observables and the dynamics of the quantum system (see, e.g., [30, Chapter X] for further results and motivation). We refer also to $[18,25,35]$ for recent textbooks that address systematically the problem of self-adjoint extension from different points of view (see also the references therein).

The question of how does the process of selecting self-adjoint extensions of symmetric operators intertwine with the notion of quantum symmetry arises. This question is at the focus of our interest in this article. We provide here natural characterizations of those self-adjoint extensions that are compatible with the given symmetries. Concretely, if a symmetric operator is $G$-invariant in the sense of Eq. (1.2), then it is clear that not all self-adjoint extensions of the operator will also be $G$-invariant. This is evident if one fixes the selfadjoint extension by selecting boundary conditions. In general, these conditions need not preserve the underlying symmetry of the system. We present in Sects. 3 and 4 the characterization of $G$-invariant self-adjoint extensions from two different point of views: first, in the most general context of deficiency spaces provided by von Neumann's theorem. Second, using the representation theorem of quadratic forms in terms of self-adjoint operators. We prove in Theorem 4.2 a $G$-invariant version of the representation theorem for quadratic forms. In Sect. 5, we give an alternative notion of $G$-invariance in terms of the theory of von Neumann algebras (cf., Proposition 5.4). We relate also here the irreducible sub-representations of $V$ with the reduction of the corresponding $G$-invariant self-adjoint extensions $T$. In particular, we show that if $T$ is unbounded and $G$-invariant, then the group $G$ must act on the Hilbert space via a highly reducible representation $V$. Finally, we apply the theory developed to a large class of self-adjoint extension of the Laplace-Beltrami operator on a smooth, compact manifold with smooth boundary on which a group is represented with a traceable unitary representation (see Definition 6.9). In particular, self-adjoint extensions of the Laplace-Beltrami operator with respect to groups acting by isometries on the manifold are discussed. In this context the extensions are labeled by suitable unitaries on the boundary of the manifold (see [19] for details). Concrete manifolds like a cylinder or a half-sphere with $\mathbf{Z}_{2}$ or $S O(2)$ actions, respectively, will also be analyzed. 
Apart from the previous considerations there are many instances where, though only partially, the previous problem has been considered. Just to mention a few here we refer to the analysis of translational symmetries and the study of self-adjoint extensions of the Laplacian in the description of a scalar quantum field in $1+1$ dimensions in a cavity [5]. In a different context, we quote the spectral analysis of Hamiltonians in concentric spherical shells where the spherical symmetry is used in a critical way $[16,17]$. In an operator theoretic context we refer, for example, to the notion of periodic Weyl-Titchmarsh functions or invariant operators with respect to linear-fractional transformations $[7,8]$. Even from a purely geometric viewpoint we should mention the analysis of isospectral manifolds in the presence of symmetries [36]. We also refer to [35, Section 13.5] for the analysis of self-adjoint extensions commuting with a conjugation.

This article is organized as follows: in Sect. 2, we summarize well-known results on the theory of self-adjoint extensions, including the theory of scales of Hilbert spaces. In the next section, we introduce the main definitions concerning $G$-invariant operators and give an explicit characterization of $G$-invariant self-adjoint extensions in the most general setting, i.e., using the abstract characterization due to von Neumann [34]. In Sect. 4, we introduce the notion of $G$-invariant quadratic forms and show that the self-adjoint operators representing them will also be $G$-invariant operators. In the following section, we present first steps of a reduction theory for $G$-invariant self-adjoint operators. For this, we use systematically the notion of an unbounded operator affiliated to a von Neumann algebra. In Sect. 6, we analyze the quadratic forms associated with the Laplace-Beltrami operator when there is a Lie group acting on the manifold. Thus, we provide a characterization of the self-adjoint extensions of the Laplace-Beltrami operator that are $G$-invariant.

Notation: In this article, all unbounded, linear operators $T$ that act on a separable, complex Hilbert space $\mathcal{H}$ are densely defined and we denote the corresponding domain by $\mathcal{D}(T) \subset \mathcal{H}$.

\section{Basic Material on Self-Adjoint Extensions}

For convenience of the reader and to fix our notation we will summarize here some standard facts on the theory of self-adjoint extensions of symmetric operators, representation theorems for quadratic forms and the theory of rigged Hilbert spaces. We refer to standard references, e.g., [3, 21, 22, 29, 35], for proofs, further details and references.

\subsection{Symmetric and Self-Adjoint Operators in Hilbert Space}

Let $T$ be an unbounded, linear operator on the complex, separable Hilbert space $\mathcal{H}$ and with dense domain $\mathcal{D}(T) \subset \mathcal{H}$. Recall that the operator $T$ is called symmetric if

$$
\langle\Psi, T \Phi\rangle=\langle T \Psi, \Phi\rangle \quad \forall \Psi, \Phi \in \mathcal{D}(T) .
$$


Moreover, $T$ is self-adjoint if it is symmetric and $\mathcal{D}(T)=\mathcal{D}\left(T^{\dagger}\right)$, where the domain of the adjoint operator $\mathcal{D}\left(T^{\dagger}\right)$ is the set of all $\Psi \in \mathcal{H}$ such that there exists $\chi \in \mathcal{H}$ with

$$
\langle\Psi, T \Phi\rangle=\langle\chi, \Phi\rangle \quad \forall \Phi \in \mathcal{D}(T) .
$$

In this case, we define $T^{\dagger} \Psi:=\chi$. If $T$ is symmetric then $T^{\dagger}$ is a closed extension of $T, T \subset T^{\dagger}$, i.e., $\mathcal{D}(T) \subset \mathcal{D}\left(T^{\dagger}\right)$ and $\left.T^{\dagger}\right|_{\mathcal{D}(T)}=T$.

The relation between self-adjoint and closed, symmetric operators is subtle and extremely important, specially from the physical point of view. It is thus natural to ask if given a symmetric operator one can find a closed extension of it that is self-adjoint and whether or not it is unique. Von Neumann addressed this issue in the late 20 s and answered the question in an abstract setting, cf., [34]. We recall the main definition and results needed later (see [30, Theorem X.2]).

Definition 2.1. Let $T$ be a closed, symmetric operator. The deficiency spaces $\mathcal{N}_{ \pm}$are defined to be

$$
\mathcal{N}_{ \pm}=\left\{\Phi \in \mathcal{H} \mid\left(T^{\dagger} \mp \mathbf{i}\right) \Phi=0\right\} .
$$

The deficiency indices are

$$
n_{ \pm}=\operatorname{dim} \mathcal{N}_{ \pm}
$$

Theorem 2.2 (von Neumann). Let $T$ be a closed, symmetric operator. The self-adjoint extensions of $T$ are in one-to-one correspondence with the set of unitaries (in the usual inner product) of $\mathcal{N}_{+}$onto $\mathcal{N}_{-}$. If $K$ is such a unitary then the corresponding self-adjoint operator $T_{K}$ has domain

$$
\mathcal{D}\left(T_{K}\right)=\left\{\Phi+(\mathbb{I}+K) \xi \mid \Phi \in \mathcal{D}(T), \quad \xi \in \mathcal{N}_{+}\right\},
$$

and

$$
T_{K}(\Phi+(\mathbb{I}+K) \xi)=T^{\dagger}(\Phi+(\mathbb{I}+K) \xi)=T \Phi+\mathbf{i}(\mathbb{I}+K) \xi .
$$

Remark 2.3. (i) The preceding definition and theorem can be also stated without assuming that the symmetric operator $T$ is closed (see, e.g., [15, Section XII.4]). In view of Corollary 3.3 and that in the context of von Neumann algebras of Sect. 5 the closure of $T$ is essential, we make this simplifying assumption here.

(ii) We refer to [28] for a recent article that characterizes the class of all selfadjoint extensions of the symmetric operator obtained as a restriction of a self-adjoint operator to a suitable subspace of its domain. In particular, the explicit relation of the techniques used to the classical result by von Neumann is also worked out.

Finally, we recall that the densely defined operator $T: \mathcal{D}(T) \rightarrow \mathcal{H}$ is semi-bounded from below if there is a constant $m \geq 0$ such that

$$
\langle\Phi, T \Phi\rangle \geq-m\|\Phi\|^{2} \quad \forall \Phi \in \mathcal{D}(T) .
$$

The operator $T$ is positive if the lower bound satisfies $m=0$. Note that semibounded operators are automatically symmetric. 


\subsection{Closable Quadratic Forms}

In this section, we introduce the notion of closed and closable quadratic forms. Standard references are, e.g., [21, Chapter VI], [29, Section VIII.6] or [13, Section 4.4].

Definition 2.4. Let $\mathcal{D}$ be a dense subspace of the Hilbert space $\mathcal{H}$ and denote by $Q: \mathcal{D} \times \mathcal{D} \rightarrow \mathbb{C}$ a sesquilinear form (anti-linear in the first entry and linear in the second entry). The quadratic form associated with $Q$ with domain $\mathcal{D}$ is its evaluation on the diagonal, i.e., $Q(\Phi):=Q(\Phi, \Phi), \Phi \in \mathcal{D}$. The sesquilinear form is called Hermitean if

$$
Q(\Phi, \Psi)=\overline{Q(\Psi, \Phi)}, \quad \Phi, \Psi \in \mathcal{D} .
$$

The quadratic form is semi-bounded from below if there is an $m \geq 0$ such that

$$
Q(\Phi) \geq-m\|\Phi\|^{2}, \quad \Phi \in \mathcal{D} .
$$

The smallest possible value $m$ satisfying the preceding inequality is called the lower bound for the quadratic form $Q$. In particular, if $Q(\Phi) \geq 0$ for all $\Phi \in \mathcal{D}$ then we call $Q$ positive.

Note that if $Q$ is semi-bounded with lower bound $m$, then

$$
Q(\Phi)+m\|\Phi\|^{2}, \quad \Phi \in \mathcal{D}
$$

is positive on the same domain. We need to recall also the notions of closable and closed quadratic forms as well as the fundamental representation theorems that relate closed, semi-bounded quadratic forms with self-adjoint, semi-bounded operators.

Definition 2.5. Let $Q$ be a semi-bounded quadratic form with lower bound $m \geq 0$ and dense domain $\mathcal{D} \subset \mathcal{H}$. The quadratic form $Q$ is closed if $\mathcal{D}$ is closed with respect to the norm

$$
\|\Phi \mid\|_{Q}:=\sqrt{Q(\Phi)+(1+m)\|\Phi\|^{2}}, \quad \Phi \in \mathcal{D} .
$$

If $\mathrm{Q}$ is closed and $\mathcal{D}_{0} \subset \mathcal{D}$ is dense with respect to the norm $\|\cdot\| \|_{Q}$, then $\mathcal{D}_{0}$ is called a form core for $Q$.

Conversely, the closed quadratic form $Q$ with domain $\mathcal{D}$ is called an extension of the quadratic form $Q$ with domain $\mathcal{D}_{0}$. A quadratic form is said to be closable if it has a closed extension.

Remark 2.6. (i) The norm $\|\cdot \cdot\|_{Q}$ is induced by the following inner product on the domain:

$$
\langle\Phi, \Psi\rangle_{Q}:=Q(\Phi, \Psi)+(1+m)\langle\Phi, \Psi\rangle, \quad \Phi, \Psi \in \mathcal{D} .
$$

(ii) It is always possible to close $\mathcal{D} \subset \mathcal{H}$ with respect to the norm $\|\cdot\| \|_{Q}$. The quadratic form is closable iff this closure is a subspace of $\mathcal{H}$.

The following representation theorem shows the deep relation between closed, semi-bounded quadratic forms and self-adjoint operators. This result goes back to the pioneering work in the 1950s by Friedrichs, Kato, Lax and Milgram, and others (see, e.g., comments to Section VIII.6 in [29]). The representation theorem can be extended to the class of sectorial forms and operators 
(see [21, Section VI.2]), but we will only need here its version for self-adjoint operators.

Theorem 2.7. Let $Q$ be an Hermitean, closed, semi-bounded quadratic form defined on the dense domain $\mathcal{D} \subset \mathcal{H}$. Then it exists a unique, self-adjoint, semi-bounded operator $T$ with domain $\mathcal{D}(T)$ and the same lower bound such that

(i) $\Psi \in \mathcal{D}(T)$ iff $\Psi \in \mathcal{D}$ and it exists $\chi \in \mathcal{H}$ such that

$$
Q(\Phi, \Psi)=\langle\Phi, \chi\rangle, \quad \forall \Phi \in \mathcal{D} .
$$

In this case, we write $T \Psi=\chi$ and $Q(\Phi, \Psi)=\langle\Phi, T \Psi\rangle$ for any $\Phi \in \mathcal{D}$, $\Psi \in \mathcal{D}(T)$.

(ii) $\mathcal{D}(T)$ is a core for $Q$.

Following [13, Theorem 4.4.2] we get the following characterization of representable quadratic forms.

Theorem 2.8. Let $Q$ be a semi-bounded, quadratic form with lower bound $m$ and domain $\mathcal{D}$. Then the following conditions are equivalent:

(i) There is a lower semi-bounded operator $T$ with lower bound $m$ representing the quadratic form $Q$.

(ii) The domain $\mathcal{D}$ is complete with respect to the norm $\|\cdot\|_{Q}$.

One of the most common uses of the representation theorem is to obtain self-adjoint extensions of symmetric, semi-bounded operators. Given a semibounded, symmetric operator $T$ one can consider the associated quadratic form

$$
Q_{T}(\Phi, \Psi)=\langle\Phi, T \Psi\rangle \quad \Phi, \Psi \in \mathcal{D}(T) .
$$

These quadratic forms are always closable, cf., [30, Theorem X.23], and, therefore, their closure is associated with a unique self-adjoint operator.

Even if a symmetric operator has uncountably many possible self-adjoint extensions, the representation theorem above allows to select a particular one given a suitable quadratic form. This extension is called the Friedrichs' or hard extension and is in a natural sense maximal (see Chapters 10 and 13 in [35] for a relation to Krein-von Neumann or soft extensions). The approach that we shall take in Sects. 4 and 6 uses this kind of Friedrichs-type extension.

\subsection{Scales of Hilbert Spaces}

The theory of scales of Hilbert spaces, also known as theory of rigged Hilbert spaces, has been used in many ways in mathematics and mathematical physics. One of the standard applications of this theory appears in the proof of the representation theorems mentioned above. We state next the main results, (see [9, Chapter I], [22, Chapter 2] for proofs and more results).

Let $\mathcal{H}$ be a Hilbert space with scalar product $\langle\cdot, \cdot\rangle$ and induced norm $\|\cdot\|$. Let $\mathcal{H}_{+}$be a dense, linear subspace of $\mathcal{H}$ which is a complete Hilbert 
space with respect to another scalar product that will be denoted by $\langle\cdot, \cdot\rangle_{+}$. The corresponding norm is $\|\cdot\|_{+}$and we assume that

$$
\|\Phi\| \leq\|\Phi\|_{+}, \quad \Phi \in \mathcal{H}_{+} .
$$

Any vector $\Phi \in \mathcal{H}$ generates a continuous linear functional $L_{\Phi}: \mathcal{H}_{+} \rightarrow \mathbb{C}$ as follows. For $\Psi \in \mathcal{H}_{+}$define

$$
L_{\Phi}(\Psi)=\langle\Phi, \Psi\rangle .
$$

Continuity follows by the Cauchy-Schwarz inequality and Eq. (2.1).

Since $L_{\Phi}$ is a continuous linear functional on $\mathcal{H}_{+}$it can be represented, according to Riesz theorem, using the scalar product in $\mathcal{H}_{+}$. Namely, it exists a vector $\xi \in \mathcal{H}_{+}$such that

$$
\forall \Psi \in \mathcal{H}_{+}, \quad L_{\Phi}(\Psi)=\langle\Phi, \Psi\rangle=\langle\xi, \Psi\rangle_{+},
$$

and the norm of the functional coincides with the norm in $\mathcal{H}_{+}$of the element $\xi$. One can use the above equalities to define an operator

$$
\begin{aligned}
\hat{I}: \mathcal{H} & \rightarrow \mathcal{H}_{+} \\
\hat{I} \Phi & =\xi .
\end{aligned}
$$

This operator is clearly injective since $\mathcal{H}_{+}$is a dense subset of $\mathcal{H}$ and therefore it can be used to define a new scalar product on $\mathcal{H}$

$$
\langle\cdot, \cdot\rangle_{-}:=\langle\hat{I} \cdot, \hat{I} \cdot\rangle_{+} \cdot
$$

The completion of $\mathcal{H}$ with respect to this scalar product defines a new Hilbert space, $\mathcal{H}_{-}$, and the corresponding norm will be denoted accordingly by $\|\cdot\|_{-}$. It is clear that $\mathcal{H}_{+} \subset \mathcal{H} \subset \mathcal{H}_{-}$, with dense inclusions. Since $\|\xi\|_{+}=\|\hat{I} \Phi\|_{+}=$ $\|\Phi\|_{-}$, the operator $\hat{I}$ can be extended by continuity to an isometric bijection.

Definition 2.9. The Hilbert spaces $\mathcal{H}_{+}, \mathcal{H}$ and $\mathcal{H}_{-}$introduced above define a scale of Hilbert spaces. The extension by continuity of the operator $\hat{I}$ is called the canonical isometric bijection. It is denoted by:

$$
I: \mathcal{H}_{-} \rightarrow \mathcal{H}_{+} .
$$

Proposition 2.10. The scalar product in $\mathcal{H}$ can be extended continuously to a pairing

$$
(\cdot, \cdot): \mathcal{H}_{-} \times \mathcal{H}_{+} \rightarrow \mathbb{C}
$$

Proof. Let $\Phi \in \mathcal{H}$ and $\Psi \in \mathcal{H}_{+}$. Using the Cauchy-Schwarz inequality we have that

$$
|\langle\Phi, \Psi\rangle|=\left|\langle I \Phi, \Psi\rangle_{+}\right| \leq\|I \Phi\|_{+}\|\Psi\|_{+}=\|\Phi\|_{-}\|\Psi\|_{+}
$$

and we can extend the scalar product by continuity to the pairing $(\cdot, \cdot)$. 


\section{Self-Adjoint Extensions with Symmetry}

We begin now analyzing the question of how the process of finding a selfadjoint extension of a symmetric operator intertwines with the notion of a quantum symmetry. We will denote by $G$ a group and let

$$
V: G \rightarrow \mathcal{U}(\mathcal{H})
$$

be a fixed unitary representation of $G$ on the complex, separable Hilbert space $\mathcal{H}$. We will introduce the notion of $G$-invariance by which we mean invariance under the fixed representation $V$.

Definition 3.1. Let $T$ be a linear operator with dense domain $\mathcal{D}(T) \subset \mathcal{H}$ and consider a unitary representation $V: G \rightarrow \mathcal{U}(\mathcal{H})$. The operator $T$ is said to be $G$-invariant if $T V(g) \supseteq V(g) T$, i.e., if $V(g) \mathcal{D}(T) \subset \mathcal{D}(T)$ for all $g \in G$ and

$$
T V(g) \Psi=V(g) T \Psi \quad \forall g \in G, \forall \Psi \in \mathcal{D}(T) .
$$

Due to the invertibility of the unitary operators representing the group we have the following immediate consequence on $G$-invariant subspaces $\mathcal{K}$ of $\mathcal{H}$ which we will use several times:

$$
\text { if } V(g) \mathcal{K} \subset \mathcal{K}, \quad \forall g \in G, \quad \text { then } V(g) \mathcal{K}=\mathcal{K} \quad \forall g \in G .
$$

Proposition 3.2. Let $T: \mathcal{D}(T) \subset \mathcal{H} \rightarrow \mathcal{H}$ be a $G$-invariant, symmetric operator Then the adjoint operator $T^{\dagger}$ is G-invariant.

Proof. Let $\Psi \in \mathcal{D}\left(T^{\dagger}\right)$. Then, according to the definition of adjoint operator there is a vector $\chi \in \mathcal{H}$ such that

$$
\langle\Psi, T \Phi\rangle=\langle\chi, \Phi\rangle \quad \forall \Phi \in \mathcal{D}(T) .
$$

Using the $G$-invariance we have

$$
\begin{aligned}
\langle V(g) \Psi, T \Phi\rangle & =\left\langle\Psi, V\left(g^{-1}\right) T \Phi\right\rangle \\
& =\left\langle\Psi, T V\left(g^{-1}\right) \Phi\right\rangle \\
& =\left\langle\chi, V\left(g^{-1}\right) \Phi\right\rangle \\
& =\langle V(g) \chi, \Phi\rangle .
\end{aligned}
$$

The preceding equalities hold for any $\Phi \in \mathcal{D}(T)$ and therefore $V(g) \Psi \in \mathcal{D}\left(T^{\dagger}\right)$. Moreover, we have that $T^{\dagger} V(g) \Psi=V(g) \chi=V(g) T^{\dagger} \Psi$.

Corollary 3.3. Let $T: \mathcal{D}(T) \subset \mathcal{H} \rightarrow \mathcal{H}$ be a $G$-invariant and symmetric operator on $\mathcal{H}$. Then its closure $\bar{T}$ is also $G$-invariant.

Proof. The operator $T$ is symmetric and, therefore, closable. From $\bar{T}=T^{\dagger \dagger}$ and since $T$ is $G$-invariant, we have by the preceding proposition that $T^{\dagger}$ is $G$-invariant, hence also $\bar{T}=\left(T^{\dagger}\right)^{\dagger}$.

The preceding result shows that we can always assume without loss of generality that the $G$-invariant symmetric operators are closed.

We begin next with the analysis of the $G$-invariance of the self-adjoint extensions given by von Neumann's classical result (cf., Theorem 2.2). 
Corollary 3.4. Let $T: \mathcal{D}(T) \subset \mathcal{H} \rightarrow \mathcal{H}$ be a closed, symmetric and $G$-invariant operator. Then, the deficiency spaces $\mathcal{N}_{ \pm}$, cf., Definition 2.1, are invariant under the action of the group, i.e.,

$$
V(g) \mathcal{N}_{ \pm}=\mathcal{N}_{ \pm}
$$

Proof. Let $\xi \in \mathcal{N}_{+} \subset \mathcal{D}\left(T^{\dagger}\right)$. Then $\left(T^{\dagger}-i\right) \xi=0$ and we have from Proposition 3.2 that

$$
\left(T^{\dagger}-i\right) V(g) \xi=V(g)\left(T^{\dagger}-i\right) \xi=0 .
$$

This shows that $V(g) \mathcal{N}_{+} \subset \mathcal{N}_{+}$for all $g \in G$ and by (3.1) we get the equality. Similarly for $\mathcal{N}_{-}$.

Theorem 3.5. Let $T: \mathcal{D}(T) \subset \mathcal{H} \rightarrow \mathcal{H}$ be a closed, symmetric and $G$-invariant operator with equal deficiency indices (cf., Definition 2.1). Let $T_{K}$ be the selfadjoint extension of $T$ defined by the unitary $K: \mathcal{N}_{+} \rightarrow \mathcal{N}_{-}$. Then $T_{K}$ is $G$-invariant iff $V(g) K \xi=K V(g) \xi$ for all $\xi \in \mathcal{N}_{+}, g \in G$.

Proof. To show the direction " $\Leftarrow$ " recall that by Theorem 2.2 the domain of $T_{K}$ is given by

$$
\mathcal{D}\left(T_{K}\right)=\mathcal{D}(T) \oplus(\mathbb{I}+K) \mathcal{N}_{+} .
$$

Let $\Psi \in \mathcal{D}(T)$ and $\xi \in \mathcal{N}_{+}$. Then we have that

$$
\begin{aligned}
V(g)(\Psi+(\mathbb{I}+K) \xi) & =V(g) \Psi+(V(g)+V(g) K) \xi \\
& =V(g) \Psi+(V(g)+K V(g)) \xi \\
& =V(g) \Psi+(\mathbb{I}+K) V(g) \xi .
\end{aligned}
$$

By assumption $V(g) \Psi \in \mathcal{D}(T)$, and by Corollary 3.4, $V(g) \xi \in \mathcal{N}_{+}$. Hence $V(g) \mathcal{D}\left(T_{K}\right) \subset \mathcal{D}\left(T_{K}\right)$. Moreover, we have that for $\Phi=\Psi+(\mathbb{I}+K) \xi \in \mathcal{D}\left(T_{K}\right)$

$$
\begin{aligned}
T_{K} V(g) \Phi & =T^{\dagger} V(g)(\Psi+(\mathbb{I}+K) \xi) \\
& =T V(g)+T^{\dagger} V(g)(\mathbb{I}+K) \xi \\
& =V(g) T \Psi+V(g) T^{\dagger}(\mathbb{I}+K) \xi=V(g) T_{K} \Phi,
\end{aligned}
$$

where we have used Proposition 3.2.

To prove the reverse implication " $\Rightarrow$ " suppose that we have the selfadjoint extension defined by the unitary

$$
K^{\prime}=V(g) K V(g)^{\dagger} .
$$

If we consider the domain $\mathcal{D}\left(T_{K^{\prime}}\right)$ defined by this unitary we have that

$$
\begin{aligned}
\mathcal{D}\left(T_{K^{\prime}}\right) & =\mathcal{D}(T)+\left(\mathbb{I}+V(g) K V(g)^{\dagger}\right) \mathcal{N}_{+} \\
& =V(g) \mathcal{D}(T)+V(g)(\mathbb{I}+K) V(g)^{\dagger} \mathcal{N}_{+} \\
& =V(g) \mathcal{D}\left(T_{K}\right)=\mathcal{D}\left(T_{K}\right),
\end{aligned}
$$

where we have used again Proposition 3.2, Corollary 3.4 and (3.1). Now von Neumann's result stated in Theorem 2.2 establishes a one-to-one correspondence between isometries $K: \mathcal{N}_{+} \rightarrow \mathcal{N}_{-}$and self-adjoint extensions of the operator $T$. Therefore $K=K^{\prime}=V(g) K V(g)^{\dagger}$ and the statement follows. 


\section{Invariant Quadratic Forms}

As mentioned in the first two sections the relation between closed, semibounded quadratic forms and self-adjoint operators is realized through the so-called representation theorems. We present here a notion of $G$-invariant quadratic form and prove a representation theorem for $G$-invariant structures.

Definition 4.1. Let $Q$ be a quadratic form with domain $\mathcal{D}$ and let $V: G \rightarrow$ $\mathcal{U}(\mathcal{H})$ be a unitary representation of the group $G$. We will say that the quadratic form is $G$-invariant if $V(g) \mathcal{D} \subset \mathcal{D}$ for all $g \in G$ and

$$
Q(V(g) \Phi)=Q(\Phi) \quad \forall \Phi \in \mathcal{D}, \forall g \in G
$$

It is clear by the polarization identity that if the quadratic form $Q$ is $G$-invariant, then the associated sesquilinear form also satisfies $Q(V(g) \Phi$, $V(g) \Psi)=Q(\Phi, \Psi), g \in G$.

We will now relate the notions of $G$-invariance for self-adjoint operators and for quadratic forms.

Theorem 4.2. Let $Q$ be a closed, semi-bounded quadratic form with domain $\mathcal{D}$ and let $T$ be the representing semi-bounded, self-adjoint operator. The quadratic form $Q$ is $G$-invariant iff the operator $T$ is $G$-invariant.

Proof. To show the direction " $\Rightarrow$ " recall from Theorem 2.7 that $\Psi \in \mathcal{D}(T)$ iff $\Psi \in \mathcal{D}$ and there exists $\chi \in \mathcal{H}$ such that

$$
Q(\Phi, \Psi)=\langle\Phi, \chi\rangle \quad \forall \Phi \in \mathcal{D} .
$$

Then, if $\Psi \in \mathcal{D}(T)$, and using the $G$-invariance of the quadratic form, we have that

$$
\begin{aligned}
Q(\Phi, V(g) \Psi) & =Q\left(V(g)^{\dagger} \Phi, \Psi\right) \\
& =\left\langle V(g)^{\dagger} \Phi, \chi\right\rangle=\langle\Phi, V(g) \chi\rangle .
\end{aligned}
$$

This implies that $V(g) \Psi \in \mathcal{D}(T)$ and from

$$
T V(g) \Psi=V(g) \chi=V(g) T \Psi, \quad \Psi \in \mathcal{D}(T), g \in G,
$$

we show the $G$-invariance of the self-adjoint operator $T$.

For the reverse implication " $\Leftarrow$ " we use the fact that $\mathcal{D}(T)$ is a core for the quadratic form. For $\Phi, \Psi \in \mathcal{D}(T)$ we have that

$$
\begin{aligned}
Q(\Phi, \Psi) & =\langle\Phi, T \Psi\rangle=\langle V(g) \Phi, V(g) T \Psi\rangle \\
& =\langle V(g) \Phi, T V(g) \Psi\rangle=Q(V(g) \Phi, V(g) \Psi) .
\end{aligned}
$$

These equalities show that the $G$-invariance of $Q$ is true at least for the elements in the domain of the operator. Now for any $\Psi \in \mathcal{D}$ there is a sequence $\left\{\Psi_{n}\right\}_{n} \subset \mathcal{D}(T)$ such that $\left\|\mid \Psi_{n}-\Psi\right\|_{Q} \rightarrow 0$. This, together with the equality above, implies that $\left\{V(g) \Psi_{n}\right\}_{n}$ is a Cauchy sequence with respect to $\|\cdot\| \|_{Q}$. Since $Q$ is closed, the limit of this sequence is in $\mathcal{D}$. Moreover, it is clear that $\lim _{n \rightarrow \infty} V(g) \Psi_{n}=V(g) \Psi$, hence

$$
\left\|V(g) \Psi_{n}-V(g) \Psi\right\|_{Q} \rightarrow 0 .
$$


So far we have proved that $V(g) \mathcal{D} \subset \mathcal{D}$. Now for any $\Phi, \Psi \in \mathcal{D}$ consider sequences $\left\{\Phi_{n}\right\}_{n},\left\{\Psi_{n}\right\}_{n} \subset \mathcal{D}(T)$ that converge, respectively, to $\Phi, \Psi \in \mathcal{D}$ in the topology induced by $\|\cdot \mid\|_{Q}$. Then the limit

$$
\begin{aligned}
Q(\Phi, \Psi) & =\lim _{n \rightarrow \infty} \lim _{m \rightarrow \infty} Q\left(\Phi_{n}, \Psi_{m}\right) \\
& =\lim _{n \rightarrow \infty} \lim _{m \rightarrow \infty} Q\left(V(g) \Phi_{n}, V(g) \Psi_{m}\right)=Q(V(g) \Phi, V(g) \Psi) .
\end{aligned}
$$

concludes the proof.

The preceding result and Theorem 2.8 allow to give the following characterization of representable $G$-invariant quadratic forms.

Proposition 4.3. Let $Q$ be a G-invariant, semi-bounded quadratic form with lower bound $m$ and domain $\mathcal{D}$. The following statements are equivalent:

(i) There is a $G$-invariant, self-adjoint operator $T$ on $\mathcal{D}(T) \subset \mathcal{H}$ with lower semi-bound $m$ and that represents the quadratic form, i.e.,

$$
Q(\Phi, \Psi)=\langle\Phi, T \Psi\rangle \quad \forall \Phi \in \mathcal{D}, \forall \Psi \in \mathcal{D}(T) .
$$

(ii) The domain $\mathcal{D}$ of the quadratic form is complete in the norm $\|\cdot\| \|_{Q}$.

To conclude this section, we make contact with the theory of scales of Hilbert spaces introduced in Sect. 2.3. Let $Q$ be a closed, semi-bounded quadratic form with domain $\mathcal{D} \subset \mathcal{H}$. We will show that if $Q$ is $G$-invariant then one can automatically produce unitary representations $V_{ \pm}$on the natural scale of Hilbert spaces

$$
\mathcal{H}_{+} \subset \mathcal{H} \subset \mathcal{H}_{-}
$$

where $\mathcal{H}_{+}:=\mathcal{D}$.

Theorem 4.4. Let $Q$ be a closed, semi-bounded, G-invariant quadratic form with lower bound $m$. Then

(i) $V$ restricts to a unitary representation $V_{+}$on $\mathcal{H}_{+}:=\mathcal{D} \subset \mathcal{H}$ with scalar product given by

$$
\langle\Phi, \Psi\rangle_{+}:=\langle\Phi, \Psi\rangle_{Q}=(1+m)\langle\Phi, \Psi\rangle+Q(\Phi, \Psi), \quad \Phi, \Psi \in \mathcal{H}_{+} .
$$

(ii) $V$ extends to a unitary representation $V_{-}$on $\mathcal{H}_{-}$and we have, on $\mathcal{H}_{-}$,

$$
V_{+}(g) I=I V_{-}(g), \quad g \in G,
$$

where $I: \mathcal{H}_{-} \rightarrow \mathcal{H}_{+}$is the canonical isometric bijection of Definition 2.9.

Proof. (i) To show that the representation $V_{+}:=\left.V\right|_{\mathcal{H}_{+}}$is unitary with respect to $\langle\cdot, \cdot\rangle_{+}$note that by definition of $G$-invariance of the quadratic form we have for any $g \in G$ that $V(g): \mathcal{H}_{+} \rightarrow \mathcal{H}_{+}$and

$$
\langle V(g) \Phi, V(g) \Psi\rangle_{+}=\langle\Phi, \Psi\rangle_{+} \quad \Phi, \Psi \in \mathcal{H}_{+} .
$$

Since any $V(g)$ is invertible we conclude that $V$ restricts to a unitary representation on $\mathcal{H}_{+}$.

(ii) To show that $V$ extends to a unitary representation $V_{-}$on $\mathcal{H}_{-}$consider first the following representation of $G$ on $\mathcal{H}_{-}$:

$$
V_{-}(g):=I^{-1} V_{+}(g) I .
$$


We show first that this representation is unitary: since $V_{-}$is invertible it is enough to check the isometry condition using part (i). Indeed, for any $\alpha, \beta \in$ $\mathcal{H}_{-}$we have

$$
\begin{aligned}
\left\langle V_{-}(g) \alpha, V_{-}(g) \beta\right\rangle_{-} & =\left\langle I^{-1} V_{+}(g) I \alpha, I^{-1} V_{+}(g) I \beta\right\rangle_{-} \\
& =\left\langle V_{+}(g) I \alpha, V_{+}(g) I \beta\right\rangle_{+}=\langle I \alpha, I \beta\rangle_{+}=\langle\alpha, \beta\rangle_{-} .
\end{aligned}
$$

The restriction of $V_{-}(g), g \in G$, to $\mathcal{H}$ coincides with $V(g)$. Indeed, consider the pairing $(\cdot, \cdot): \mathcal{H}_{-} \times \mathcal{H}_{+} \rightarrow \mathbb{C}$ of Proposition 2.10 and let $\Phi \in \mathcal{H} \subset \mathcal{H}_{-}$. Then for all $\Psi \in \mathcal{H}_{+}$

$$
\begin{aligned}
\left(V_{-}(g) \Phi, \Psi\right) & =\left(I^{-1} V_{+}(g) I \Phi, \Psi\right) \\
& =\left\langle V(g)_{+} I \Phi, \Psi\right\rangle_{+} \\
& =\left\langle I \Phi, V_{+}\left(g^{-1}\right) \Psi\right\rangle_{+} \\
& =\left(\Phi, V_{+}\left(g^{-1}\right) \Psi\right) \\
& =\left\langle\Phi, V\left(g^{-1}\right) \Psi\right\rangle \\
& =\langle V(g) \Phi, \Psi\rangle=(V(g) \Phi, \Psi) .
\end{aligned}
$$

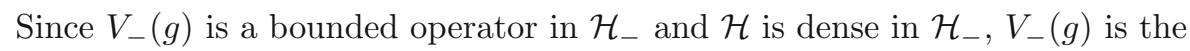
extension of $V(g)$ to $\mathcal{H}_{-}$.

\section{Reduction Theory}

The aim of this section is to provide an alternative point of view for the notion of $G$-invariance of operators (cf., Sect. 3) in terms of von Neumann algebras. Based on this approach we will address some reduction issues of the unbounded operator in terms of the reducibility of unitary representation $V$ implementing the quantum symmetry.

Recall that a von Neumann algebra $\mathcal{A}$ is a unital *-subalgebra of $\mathcal{L}(\mathcal{H})$ (the set of bounded linear operators in $\mathcal{H}$ ) which is closed in the weak operator topology. Even if a von Neumann algebra consists only of bounded linear operators acting on a Hilbert space, this class of operator algebras can be related in a natural way to closed, unbounded and densely defined operators. In fact, von Neumann introduced these algebras in 1929 and proved the celebrated bicommutant theorem, when he extended the spectral theorem to closed, unbounded normal operators in a Hilbert space (cf., [33]). Since then, the notion of affiliation of an unbounded operator to an operator algebra has been applied in different situations (see, e.g., $[10,41,42]$ ).

Let $\mathcal{S}$ be a self-adjoint subset of $\mathcal{L}(\mathcal{H})$, i.e., if $S \in \mathcal{S}$, then $S^{*} \in \mathcal{S}$. We denote by $\mathcal{S}^{\prime}$ the commutant of $\mathcal{S}$ in $\mathcal{L}(\mathcal{H})$, i.e., the set of all bounded and linear operators on $\mathcal{H}$ commuting with all operators in $\mathcal{S}$. It is a fact that $\mathcal{S}^{\prime}$ is a von Neumann algebra and that the corresponding bicommutant $\mathcal{S}^{\prime \prime}:=\left(\mathcal{S}^{\prime}\right)^{\prime}$ is the smallest von Neumann algebra containing $\mathcal{S}$, i.e., $\mathcal{S}^{\prime \prime}$ is the von Neumann algebra generated by the set $\mathcal{S} \subset \mathcal{L}(\mathcal{H})$. We refer to Sections 4.6 and 5.2 of [27] for further details and proofs. 
The definition of commutant of a densely defined unbounded operator $T$ is more delicate since one has to take into account the domains. The following definition generalizes the notion of commutant mentioned before.

Definition 5.1. Let $T: \mathcal{D}(T) \subset \mathcal{H} \rightarrow \mathcal{H}$ be a closed, densely defined operator. The commutant of $T$ is given by

$$
\{T\}^{\prime}:=\{A \in \mathcal{L}(\mathcal{H}) \mid A \mathcal{D}(T) \subset \mathcal{D}(T) \quad \text { and } \quad T A \Phi=A T \Phi, \Phi \in \mathcal{D}(T)\},
$$

i.e., $A \in\{T\}^{\prime}$ if $T A \supseteq A T$.

Since $T$ is a closed operator we have that $\{T\}^{\prime} \cap\left\{T^{\dagger}\right\}^{\prime}$ is a von Neumann algebra in $\mathcal{L}(\mathcal{H})$. We denote the von Neumann algebra associated with the bounded components of $T$ as

$$
W^{*}(T):=\left(\{T\}^{\prime} \cap\left\{T^{\dagger}\right\}^{\prime}\right)^{\prime} \subset \mathcal{L}(\mathcal{H}) .
$$

In particular, if $T$ is self-adjoint, the spectral projections of $T$ are contained in $W^{*}(T)$.

Definition 5.2. The closed, densely defined operator $T: \mathcal{D}(T) \subset \mathcal{H} \rightarrow \mathcal{H}$ is affiliated to a von Neumann algebra $\mathcal{A} \subset \mathcal{L}(\mathcal{H})$ (and we write this as $\operatorname{Ta} \mathcal{A}$ ) if

$$
W^{*}(T) \subset \mathcal{A} \text {. }
$$

Remark 5.3. There are different equivalent characterizations of the notion of affiliation: $T a \mathcal{A}$ iff $\{T\}^{\prime} \cap\left\{T^{\dagger}\right\}^{\prime} \supset \mathcal{A}^{\prime}$. In particular, this implies that $T A^{\prime} \supseteq$ $A^{\prime} T, A^{\prime} \in \mathcal{A}^{\prime}$, i.e., $A^{\prime} \mathcal{D}(T) \subset \mathcal{D}(T)$ and $T A^{\prime} \Phi=A^{\prime} T \Phi$ for all $A^{\prime} \in \mathcal{A}^{\prime}$, $\Phi \in \mathcal{D}(T)$. If $T$ is an (unbounded) self-adjoint operator, then $W^{*}(T)$ coincides with the von Neumann algebra $\mathcal{C}(T)^{\prime \prime}$ generated by the Cayley transform of $T$. Recall that the Cayley transform

$$
\mathcal{C}(T):=(\mathbb{I}-i T)(\mathbb{I}+i T)^{-1} \in \mathcal{U}(\mathcal{H})
$$

is a unitary that can be associated with $T$. We conclude that $\operatorname{Ta} \mathcal{A}$ iff $\mathcal{C}(T) \in \mathcal{A}$.

Finally, note that if $T$ is a bounded operator then, $W^{*}(T)=\left\{T, T^{\dagger}\right\}^{\prime \prime}$ is the von Neumann algebra generated by $T, T^{\dagger}$ and $T a \mathcal{A}$ iff $T, T^{\dagger} \in \mathcal{A}$.

In the following result, we will give a useful characterization of $G$ invariance for symmetric operators in terms of the affiliation to the commutant of the quantum symmetry.

Proposition 5.4. Let $T: \mathcal{D}(T) \subset \mathcal{H} \rightarrow \mathcal{H}$ be a closed, symmetric operator. Then, $T$ is G-invariant iff $T a \mathcal{V}^{\prime}$, where $\mathcal{V}$ is the von Neumann algebra generated by $\{V(g) \mid g \in G\}$ (i.e., $\mathcal{V}=\{V(g) \mid g \in G\}^{\prime \prime} \subset \mathcal{L}(\mathcal{H})$ ) and $\mathcal{V}^{\prime}$ its commutant. Moreover, any $G$-invariant self-adjoint extension of $T$ is also affiliated to $\mathcal{V}^{\prime}$.

Proof. If $T a \mathcal{V}^{\prime}$, then it is immediate that $T$ is $G$-invariant, since $\left(\mathcal{V}^{\prime}\right)^{\prime} \subset\{T\}^{\prime} \cap$ $\left\{T^{\dagger}\right\}^{\prime}$ and therefore the generators $\{V(g) \mid g \in G\}$ of the von Neumann algebra $\mathcal{V}=\mathcal{V}^{\prime \prime}$ satisfy $\{V(g) \mid g \in G\} \subset\{T\}^{\prime}$. This gives the $G$-invariance of $T$ (cf., Definition 5.1). 
To show the reverse implication assume that $T$ is $G$-invariant according to Definition 3.1, i.e., $\{V(g) \mid g \in G\} \subset\{T\}^{\prime}$. From Proposition 3.2, we also have that

$$
\{V(g) \mid g \in G\} \subset\{T\}^{\prime} \cap\left\{T^{\dagger}\right\}^{\prime}
$$

hence

$$
\mathcal{V}^{\prime}=\{V(g) \mid g \in G\}^{\prime} \supset\left(\{T\}^{\prime} \cap\left\{T^{\dagger}\right\}^{\prime}\right)^{\prime}=W^{*}(T),
$$

which implies that $T a \mathcal{V}^{\prime}$. The same argument shows that any $G$-invariant, self-adjoint extension of $T$ is also affiliated to $\mathcal{V}^{\prime}$.

We begin next with the analysis of the relation between the reducing subspaces of the quantum symmetry $V$ and those of the self-adjoint operator $T$ defined on the dense domain $\mathcal{D}(T)$. The following result and part (ii) of Theorem 5.7 are straightforward consequences of Schur's lemma.

Lemma 5.5. Let $T: \mathcal{D}(T) \subset \mathcal{H} \rightarrow \mathcal{H}$ be a self-adjoint operator. If $T$ is $G$ invariant with respect to a unitary, irreducible representation $V$ of $G$ on the Hilbert space $\mathcal{H}$, then $T$ must be bounded and

$$
T=i\left(\frac{\lambda-1}{\lambda+1}\right) \mathbb{I} \quad \text { for some } \quad \lambda \in \mathbb{T} \backslash\{-1\} .
$$

Proof. Schur's lemma and the irreducibility of $V$ imply that $\mathcal{V}^{\prime}=\mathbb{C I}$. Moreover, by Proposition 5.4 and since the Cayley transform is a unitary and $\mathcal{C}(T) \in \mathcal{V}^{\prime}$ we have

$$
\mathcal{C}(T)=(\mathbb{I}-i T)(\mathbb{I}+i T)^{-1}=\lambda \mathbb{I} \quad \text { for some } \quad \lambda \in \mathbb{T} .
$$

The case $\lambda=-1$ is not possible since $\mathcal{C}(T)$ is an isometry of $(\mathbb{I}+i T) \mathcal{D}(T)$ onto $(\mathbb{I}-i T) \mathcal{D}(T)$ and $\mathcal{D}(T)$ is dense. Therefore $\mathcal{C}(T)=\lambda \mathbb{I}$ for some $\lambda \in \mathbb{T} \backslash\{-1\}$. This implies that for any $\Phi \in \mathcal{D}(T)$ we have

$$
T \Phi=i\left(\frac{\lambda-1}{\lambda+1}\right) \Phi .
$$

Since the right-hand side of the previous equation is bounded we can extend the formula for $T$ to the whole Hilbert space.

To continue our analysis, we have to define first in which sense an unbounded operator can be reduced by a closed subspace. Roughly speaking, the reduction means that we can write $T$ as the sum of two parts: one acting on the reducing subspace and one acting on its orthogonal complement. The following definition generalizes the standard one for bounded operators and uses the notion of commutant of an unbounded operator as in Definition 5.1.

Definition 5.6. Let $T: \mathcal{D}(T) \subset \mathcal{H} \rightarrow \mathcal{H}$ be a self-adjoint operator and $\mathcal{H}_{1}$ be a closed subspace of the Hilbert space $\mathcal{H}$. We denote by $P_{1}$ the orthogonal projection onto $\mathcal{H}_{1}$. The subspace $\mathcal{H}_{1}$ (or $P_{1}$ ) reduces $T$ if $P_{1} \in\{T\}^{\prime}$, i.e., if $P_{1} \mathcal{D}(T) \subset \mathcal{D}(T)$ and $T P_{1} \Phi=P_{1} T \Phi, \Phi \in \mathcal{D}(T)$. 
The previous definition implies that if $\mathcal{H}_{1}$ is reducing for $T$, then $P_{1}^{\perp}=$ $\mathbb{I}-P_{1}$ is also reducing and $\mathcal{D}(T)=\mathcal{D}(T) \cap \mathcal{H}_{1}+\mathcal{D}(T) \cap \mathcal{H}_{1}^{\perp}$. Moreover, the subspace $\mathcal{H}_{1}$ is invariant in the sense that

$$
T \mid\left(\mathcal{D}(T) \cap \mathcal{H}_{1}\right) \subset \mathcal{H}_{1} \quad \text { and } \quad T \mid\left(\mathcal{D}(T) \cap \mathcal{H}_{1}^{\perp}\right) \subset \mathcal{H}_{1}^{\perp} .
$$

If $T$ is self-adjoint, then the spectral projections $E(\omega)$ (with $\omega$ Borel on the spectrum $\sigma(T))$ reduce $T$.

Theorem 5.7. Let $G$ be a group and consider a unitary, reducible representation $V$ which decomposes as

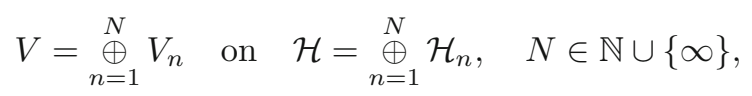

where the sub-representations $V_{k}, k=1, \ldots, N$ are irreducible and mutually inequivalent. Let $T: \mathcal{D}(T) \subset \mathcal{H} \rightarrow \mathcal{H}$ be a self-adjoint and $G$-invariant operator with respect to the representation $V$. Then

(i) Any projection $P_{k}$ onto $\mathcal{H}_{k}, k=1, \ldots, N$, is central in $\mathcal{V}$, (i.e., $P_{k} \in$ $\mathcal{V} \cap \mathcal{V}^{\prime}$ ), and reduces the operator $T$, (i.e., $P_{k} \in\{T\}^{\prime}$ ).

(ii) If $N<\infty$, then $T$ must be a bounded operator and there exist $\lambda_{k} \in$ $\mathbb{T} \backslash\{-1\}, k=1, \ldots, N$, such that

$$
T \cong i \operatorname{diag}\left(\left(\frac{\lambda_{1}-1}{\lambda_{1}+1}\right) \mathbb{I}_{\mathcal{H}_{1}}, \ldots,\left(\frac{\lambda_{N}-1}{\lambda_{N}+1}\right) \mathbb{I}_{\mathcal{H}_{N}}\right) .
$$

Proof. (i) Since the $V_{k}$ 's are all irreducible and mutually inequivalent it follows by Schur's lemma that

$$
\mathcal{V}^{\prime} \cong\left\{\operatorname{diag}\left(\lambda_{1} \mathbb{I}_{\mathcal{H}_{1}}, \ldots, \lambda_{N} \mathbb{I}_{\mathcal{H}_{N}}\right) \mid \lambda_{1}, \ldots, \lambda_{N} \in \mathbb{C}\right\} .
$$

Moreover, since any $P_{k}$ reduces $V$ it is immediate that the projections are central. To show that $P_{k} \in\{T\}^{\prime}, k=1, \ldots, N$, consider the spectral projections $E(\cdot)$ of $T$ and define for any $\Phi \in \mathcal{D}(T)$ the following positive finite measure on the Borel sets of $\sigma(T)$ :

$$
\mu_{\Phi}(\omega):=\|E(\omega) \Phi\|^{2}=\langle\Phi, E(\omega) \Phi\rangle .
$$

Now, any $P_{k}$ is central for $\mathcal{V}$ and, by $G$-invariance, Proposition 5.4 implies that $E(\omega) \in \mathcal{V}^{\prime}$ for any Borel set $\omega$. Therefore we have

$$
\mu_{P_{k} \Phi}(\omega)=\left\|E(\omega) P_{k} \Phi\right\|^{2} \leq\|E(\omega) \Phi\|^{2}=\mu_{\Phi}(\omega)
$$

and this implies that $P_{k} \mathcal{D}(T) \subset \mathcal{D}(T)$. Similarly, using the spectral theorem one can show that

$$
\left\langle y, T P_{k} \Phi\right\rangle=\left\langle y, P_{k} T \Phi\right\rangle \text { for all } y \in \mathcal{H}, \Phi \in \mathcal{D}(T),
$$

hence $P_{k} \in\{T\}^{\prime}$.

(ii) Since $T=T^{*}$ we have that the Cayley transform is unitary and

$$
\mathcal{C}(T)=(\mathbb{I}-i T)(\mathbb{I}+i T)^{-1} \in \mathcal{V}^{\prime} .
$$

Therefore, there is a $\lambda_{k} \in \mathbb{T}, k=1, \ldots, N$, such that

$$
\mathcal{C}(T) \cong \operatorname{diag}\left(\lambda_{1} \mathbb{I}_{\mathcal{H}_{1}}, \ldots, \lambda_{N} \mathbb{I}_{\mathcal{H}_{N}}\right) .
$$


As in the proof of Lemma 5.5 we exclude first the case $\lambda_{k}=-1, k=1, \ldots, N$. If $\lambda_{k}=-1$ and since the projection $P_{k}$ is reducing we have for any $\Phi_{k} \in P_{k} \mathcal{D}(T)$ that

$$
(\mathbb{I}+i T) \Phi_{k}=-(\mathbb{I}-i T) \Phi_{k} \quad \Rightarrow \quad \Phi_{k}=0 .
$$

Therefore $P_{k} \mathcal{D}(T)=\{0\}$ and we can omit the $k$ th-summand in the decomposition of $T$. Hence without loss of generality we can assume that $\lambda_{k} \in \mathbb{T} \backslash\{-1\}$, $k=1, \ldots, N$ and a similar reasoning on each block as in Lemma 5.5 gives the formula for $T$.

Part (ii) of the previous theorem says that any representation of $V$ implementing a quantum symmetry of an unbounded, self-adjoint operator must be highly reducible. Note that only if $N=\infty$ may $T$ be unbounded. E.g., consider the case where $\lim _{N} \lambda_{N}=-1$. In the particular case of a compact group acting on an infinite-dimensional Hilbert space, we know that the decomposition of $V$ into irreducible representations must have infinite irreducible components. In this sense, the representations considered in the examples of the following sections are meaningful. The following remark and Proposition 5.9 show that this is so even if we consider equivalent irreducible representations.

Remark 5.8. If the irreducible representations are not mutually inequivalent, then the corresponding projections need not be reducing. In fact, consider the example $V=V_{1} \oplus V_{2}$ on $\mathcal{H}=\mathcal{H}_{1} \oplus \mathcal{H}_{2}$ with $V_{1} \cong V_{2}$, i.e., there is a unitary $U: \mathcal{H}_{1} \rightarrow \mathcal{H}_{2}$ such that $V_{2}=U V_{1} U^{*}$. Then

$$
\mathcal{V}^{\prime}=\left\{\left(\begin{array}{cc}
\lambda_{1} \mathbb{I}_{\mathcal{H}_{1}} & \lambda_{2} U^{*} \\
\lambda_{3} U & \lambda_{4} \mathbb{I}_{\mathcal{H}_{2}}
\end{array}\right) \mid \lambda_{k} \in \mathbb{C}, k=1, \ldots, 4\right\}
$$

and

$$
\mathcal{V}=\mathcal{V}^{\prime \prime}=\left\{\left(\begin{array}{cc}
A_{1} & 0 \\
0 & U A_{1} U^{*}
\end{array}\right) \mid A_{1} \in \mathcal{L}\left(\mathcal{H}_{1}\right)\right\} .
$$

This shows that $P_{k} \notin \mathcal{V}$ and, in fact if $V$ is a quantum symmetry for $T$, then $P_{k}$ need not be reducing for $T$.

It can be shown that $T$ must also be bounded in this later case. Take into account that it is not assumed that the irreducible representations are finite dimensional. Below we show this in the simple case that the representation $V$ is a composition of two equivalent representations. The generalization to a finite number of equivalent representations is straightforward.

Proposition 5.9. Let $G$ be a group and consider a unitary, reducible representation $V$ which decomposes as a direct sum of two equivalent, irreducible representations. Let $T: \mathcal{D}(T) \subset \mathcal{H} \rightarrow \mathcal{H}$ be a $G$-invariant, self-adjoint operator with respect to the representation $V$. Then $T$ must be a bounded operator.

Proof. By assumption, we have that $V=V_{1} \oplus V_{2}$ with $V_{2}=U V_{1} U^{*}$ where $U: \mathcal{H}_{1} \rightarrow \mathcal{H}_{2}$ is the unitary operator representing the equivalence and $\mathcal{H}=$ 
$\mathcal{H}_{1} \oplus \mathcal{H}_{2}$. According to the previous remark we have that the Cayley transform of the operator $T$ is

$$
\mathcal{C}(T)=\left(\begin{array}{cc}
\lambda_{1} \mathbb{I}_{\mathcal{H}_{1}} & \lambda_{2} U^{*} \\
\lambda_{3} U & \lambda_{4} \mathbb{I}_{\mathcal{H}_{2}}
\end{array}\right), \quad \text { for some } \quad \lambda_{k} \in \mathbb{C}, k=1, \ldots, 4
$$

Moreover, since $\mathcal{C}(T)$ is a unitary operator, the coefficient matrix

$$
\Lambda:=\left(\begin{array}{ll}
\lambda_{1} & \lambda_{2} \\
\lambda_{3} & \lambda_{4}
\end{array}\right)
$$

is a $2 \times 2$ unitary matrix, i.e., $\Lambda \in \mathcal{U}(2)$. Therefore it exists a unique, unitary matrix

$$
\Sigma=\left(\begin{array}{ll}
s_{1} & s_{2} \\
s_{3} & s_{4}
\end{array}\right) \in \mathcal{U}(2)
$$

that diagonalizes $\Lambda$, i.e., $\Sigma^{*} \Lambda \Sigma=\operatorname{diag}\left(\tilde{\lambda}_{1}, \tilde{\lambda}_{2}\right), \tilde{\lambda}_{k} \in \mathbb{T}, k=1$, 2 . Consider the unitary operator

$$
S=\left(\begin{array}{cc}
s_{1} \mathbb{I}_{\mathcal{H}_{1}} & s_{2} U^{*} \\
s_{3} U & s_{4} \mathbb{I}_{\mathcal{H}_{2}}
\end{array}\right)
$$

This unitary transformation satisfies that

$$
S^{*} \mathcal{C}(T) S=\begin{array}{cc}
\tilde{\lambda}_{1} \mathbb{I}_{\tilde{\mathcal{H}}_{1}} & 0 \\
0 & \tilde{\lambda}_{2} \mathbb{I}_{\tilde{\mathcal{H}}_{2}}
\end{array} \quad \text { for some } \quad \tilde{\lambda}_{1}, \tilde{\lambda}_{2} \in \mathbb{T}
$$

where the new block structure represents a different decomposition of $\mathcal{H}=$ $\tilde{\mathcal{H}}_{1} \oplus \tilde{\mathcal{H}}_{2}$. With respect to this decomposition there are associated two proper subspaces of $\mathcal{C}(T)$ with proper projections $\tilde{P}_{1}$ and $\tilde{P}_{2}$. Notice that these projections reduce $T$. These projections do not coincide in general with $P_{1}$ and $P_{2}$, the projections associated with the decomposition $V=V_{1} \oplus V_{2}$.

The same arguments as in the proof of Theorem 5.7 lead us to exclude the cases $\tilde{\lambda}_{k}=-1$ and we can consider that $\tilde{\lambda}_{k} \in \mathbb{T} \backslash\{-1\}, k=1,2$. Hence $T$ is a bounded operator.

The following result is a straightforward consequence of the main results in this section.

Corollary 5.10. Let $T$ be an unbounded self-adjoint operator on a Hilbert space $\mathcal{H}$ which is $G$-invariant with respect to a unitary representation $V: G \rightarrow \mathcal{U}(\mathcal{H})$. Then $V$ cannot be a direct sum of finitely many irreducible representations.

The present section is a first step to analyze the relation between the reduction theory of a quantum mechanical symmetry and the reduction of the unbounded $G$-invariant operator. In particular, we consider only when $V$ decomposes as a direct sum of irreducible representations. This is enough for the applications we have in mind in the following sections, where mainly compact groups act as a quantum symmetry. For a systematic and general theory of reduction one has to address, among other things, the type decomposition of the von Neumann algebras corresponding to the intertwiner spaces of the representation $V$ and the corresponding direct integral decomposition of the self-adjoint operator $T$ (see, e.g., $[24,26]$ ). 


\section{Invariant Self-Adjoint Extensions of the Laplace-Beltrami Operator}

As an application of the previous results we analyze the class of self-adjoint extensions of the Laplace-Beltrami operator on a Riemannian manifold introduced in [19] according to their invariance properties with respect to a symmetry group, in particular with respect to a group action on the manifold.

Throughout the rest of this and the next section we will consider a smooth, compact, Riemannian manifold with boundary $(\Omega, \partial \Omega, \eta)$. The boundary $\partial \Omega$ of the Riemannian manifold $(\Omega, \partial \Omega, \eta)$ has itself the structure of a Riemannian manifold without boundary $(\partial \Omega, \partial \eta)$. The Riemannian metric at the boundary is just the pull-back of the Riemannian metric $\partial \eta=i^{*} \eta$, where $i: \partial \Omega \hookrightarrow \Omega$ is the canonical inclusion map. The spaces of smooth functions over the manifolds verify that

$$
\left.\mathcal{C}^{\infty}(\Omega)\right|_{\partial \Omega} \simeq \mathcal{C}^{\infty}(\partial \Omega)
$$

The Sobolev spaces of order $k \in \mathbb{R}^{+}$over the manifolds $(\Omega, \partial \Omega, \eta)$ and $(\partial \Omega, \partial \eta)$ are going to be denoted by $\mathcal{H}^{k}(\Omega)$ and $\mathcal{H}^{k}(\partial \Omega)$, respectively. There is an important relation between the Sobolev spaces defined over the manifolds $\Omega$ and $\partial \Omega$. This is the well-known Lions trace theorem (see, e.g., [2, Theorem 7.39] and Theorem 9.4 of Chapter 1 in [23]).

Theorem 6.1 (Lions). Let $\Phi \in \mathcal{C}^{\infty}(\Omega)$ and let $\gamma: \mathcal{C}^{\infty}(\Omega) \rightarrow \mathcal{C}^{\infty}(\partial \Omega)$ be the trace map $\gamma(\Phi)=\left.\Phi\right|_{\partial \Omega}$. There is a unique continuous extension of the trace map such that

(i) $\gamma: \mathcal{H}^{k}(\Omega) \rightarrow \mathcal{H}^{k-1 / 2}(\partial \Omega), k>1 / 2$.

(ii) The map is surjective.

\subsection{A Class of Self-Adjoint Extensions of the Laplace-Beltrami Operator}

We recall here some results from [19] that describe a large class of self-adjoint extensions of the Laplace-Beltrami operator. The extensions are parameterized in terms of suitable unitaries on the boundary Hilbert space. This class is constructed in terms of a family of closed, semi-bounded quadratic forms via the representation theorem (cf., Theorem 2.7). Before introducing this family we shall need some definitions.

Definition 6.2. Let $U: \mathcal{L}^{2}(\partial \Omega) \rightarrow \mathcal{L}^{2}(\partial \Omega)$ be unitary and denote by $\sigma(U)$ its spectrum. The unitary $U$ on the boundary has spectral gap at -1 if one of the following conditions hold:

(i) $\mathbb{I}+U$ is invertible.

(ii) $-1 \in \sigma(U)$ and -1 is not an accumulation point of $\sigma(U)$.

The eigenspace associated with the eigenvalue -1 is denoted by $W$. The corresponding orthogonal projections will be written as $P_{W}$ and $P_{W^{\perp}}=\mathbb{I}-P_{W}$.

Definition 6.3. Let $U$ be a unitary operator acting on $\mathcal{L}^{2}(\partial \Omega)$ with spectral gap at -1 . The partial Cayley transform $A_{U}: \mathcal{L}^{2}(\partial \Omega) \rightarrow \mathcal{L}^{2}(\partial \Omega)$ is the operator

$$
A_{U}:=\mathbf{i} P_{W^{\perp}}(U-\mathbb{I})(U+\mathbb{I})^{-1} \text {. }
$$


Definition 6.4. Let $U$ be a unitary with spectral gap at -1 . The unitary is said to be admissible if the partial Cayley transform $A_{U}$ leaves the subspace $\mathcal{H}^{1 / 2}(\partial \Omega)$ invariant and is continuous with respect to the Sobolev norm of order $1 / 2$, i.e.,

$$
\left\|A_{U} \varphi\right\|_{\mathcal{H}^{1 / 2}(\partial \Omega)} \leq K\|\varphi\|_{\mathcal{H}^{1 / 2}(\partial \Omega)}
$$

Example 6.5. Consider a manifold with boundary given by the unit circle, i.e., $\partial \Omega=S^{1}$, and define the unitary $\left(U_{\beta} \varphi\right)(z):=e^{i \beta(z)} \varphi(z), \varphi \in \mathcal{L}^{2}\left(S^{1}\right)$. If $\beta \in \mathcal{L}^{2}\left(S^{1}\right)$ and $\operatorname{ran} \beta \subset\{\pi\} \cup[0, \pi-\delta] \cup[\pi+\delta, 2 \pi)$, for some $\delta>0$, then $U_{\beta}$ has gap at -1 . If, in addition, $\beta \in C^{\infty}\left(S^{1}\right)$, then $U_{\beta}$ is admissible.

Definition 6.6. Let $U$ be a unitary with spectral gap at $-1, A_{U}$ the corresponding partial Cayley transform and $\gamma$ the trace map considered in Theorem 6.1. The Hermitean quadratic form $Q_{U}$ associated with the unitary $U$ is defined by

$$
Q_{U}(\Phi, \Psi)=\langle\mathrm{d} \Phi, \mathrm{d} \Psi\rangle_{\Lambda^{1}}-\left\langle\gamma(\Phi), A_{U} \gamma(\Phi)\right\rangle_{\partial \Omega}
$$

on the domain

$$
\mathcal{D}_{U}=\left\{\Phi \in \mathcal{H}^{1}(\Omega) \mid P_{W} \gamma(\Phi)=0\right\} .
$$

Here $\langle\cdot, \cdot\rangle_{\Lambda^{1}}$ stands for the canonical scalar product among one-forms on the manifold $\Omega$.

It is worth to mention the reasons behind Definitions 6.2 and 6.4. The spectral gap condition ensures that the partial Cayley transform becomes a bounded, self-adjoint operator on the subspace $W^{\perp}$ and this guarantees that the quadratic form $Q_{U}$ is lower semi-bounded. Notice that we are dealing with unbounded quadratic forms and thus they are not continuous mappings of the Hilbert space. The admissibility condition is an analytic requirement to ensure that $Q_{U}$ is a closable quadratic form.

In the next theorem, we give a class of self-adjoint extensions of the minimal Laplacian operator $\Delta_{\text {min }}$ on the domain $\mathcal{H}_{0}^{2}(\Omega)$. We refer to [19, Section 4$]$ for a complete proof and additional motivation. All the extensions are labeled by suitable unitaries $U$ at the boundary.

Theorem 6.7. Let $U: \mathcal{L}^{2}(\partial \Omega) \rightarrow \mathcal{L}^{2}(\partial \Omega)$ be an admissible unitary operator with spectral gap at -1 . Then the quadratic form $Q_{U}$ of Definition 6.6 is semibounded from below and closable. Its closure is represented by a semi-bounded, self-adjoint extension of the minimal Laplacian $-\Delta_{\min }$.

\subsection{Unitaries at the Boundary and $G$-Invariance}

We will use next the results of Sect. 4 to give necessary and sufficient conditions on the characterization of the unitary $U$ in order that the corresponding quadratic form $\left(Q_{U}, \mathcal{D}_{U}\right)$ is $G$-invariant. In particular, from Theorem 4.2, we conclude that the self-adjoint operator representing its closure will also be $G$-invariant.

We need to consider first the quadratic form corresponding to the Neumann extension of the Laplace-Beltrami operator:

$$
Q_{N}(\Phi)=\|d \Phi\|^{2}, \quad \Phi \in \mathcal{D}_{N}=\mathcal{H}^{1}(\Omega) .
$$


We will also call it Neumann quadratic form. Note that it corresponds to the quadratic form $Q_{U}$ of the previous section with admissible unitary $U=\mathbb{I}$. Moreover, $U$ has spectral gap at -1 and for the corresponding orthogonal projection we have $P_{W}=\mathbb{I}$, hence $A_{U}=0$.

Let $G$ be a Lie group and $V: G \rightarrow \mathcal{U}\left(\mathcal{L}^{2}(\Omega)\right)$ be a continuous unitary representation of $G$, i.e., for any $\Phi \in \mathcal{L}^{2}(\Omega)$ the map

$$
G \ni g \mapsto V(g) \Phi
$$

is continuous in the $\mathcal{L}^{2}$-norm. We will assume that $Q_{N}$ is $G$-invariant, that is, $V(g) \mathcal{H}^{1}(\Omega) \subset \mathcal{H}^{1}(\Omega)$ and $Q_{N}(V(g) \Phi)=Q_{N}(\Phi)$ for all $\Phi \in \mathcal{H}^{1}(\Omega)$ and $g \in G$. Then the following lemma shows that $V$ defines also a continuous unitary representation on $\mathcal{H}^{1}(\Omega)$ with its corresponding Sobolev scalar product.

Lemma 6.8. Let $V$ a strongly continuous unitary representation of the Lie group $G$ on $\mathcal{L}^{2}(\Omega)$ such that Neumann's quadratic form $Q_{N}$ is $G$-invariant. Then $V$ leaves invariant the subspace $\mathcal{H}^{1}(\Omega)$ and defines a strongly continuous unitary representation on it.

Proof. Since $V(g)$ is invertible it is enough to show that $V(g)$ is an isometry with respect to the Sobolev norm $\|\cdot\|_{1}$ (see also the proof of Theorem 4.4). But this is immediate since $V$ is unitary on $\mathcal{L}^{2}(\Omega)$ and $Q_{N}$ is $G$-invariant. This is trivial if we realize that $\|\cdot\|_{1}^{2}=\|\cdot\|_{0}^{2}+Q_{N}(\cdot)$, then because of the $G$-invariance of $Q_{N}$ we get that $\|V(g) \Phi\|_{1}=\|\Phi\|_{1}$ for all $\Phi \in \mathcal{H}^{1}(\Omega), g \in G$.

Finally, to prove strong continuity on $\mathcal{H}^{1}(\Omega)$, use the invariance

$$
\|V(g) \Phi\|_{1}=\|\Phi\|_{1}, \quad g \in G
$$

and a standard weak compactness argument.

Definition 6.9. The representation $V: G \rightarrow \mathcal{L}^{2}(\Omega)$ has a trace (or that it is traceable) along the boundary $\partial \Omega$ if there exists another continuous, unitary representation $v: G \rightarrow \mathcal{U}\left(\mathcal{L}^{2}(\partial \Omega)\right)$ such that

$$
\gamma(V(g) \Phi)=v(g) \gamma(\Phi),
$$

for all $\Phi \in \mathcal{H}^{1}(\Omega)$ and $g \in G$ or, in other words, that the following diagram is commutative:

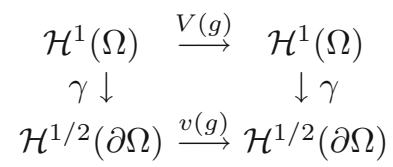

We will call $v$ the trace of the representation $V$.

Notice that if the representation $V$ is traceable, its trace $v$ is unique.

Now we are able to prove the following theorem:

Theorem 6.10. Let $G$ a Lie group, and $V: G \rightarrow \mathcal{U}\left(\mathcal{L}^{2}(\Omega)\right)$ a traceable continuous, unitary representation of $G$ with unitary trace $v: G \rightarrow \mathcal{U}\left(\mathcal{L}^{2}(\partial \Omega)\right)$ along the boundary $\partial \Omega$. Denote by $\left(Q_{U}, \mathcal{D}_{U}\right)$ the closable and semi-bounded quadratic form of Definition 6.6 with an admissible unitary $U \in \mathcal{U}\left(\mathcal{L}^{2}(\Omega)\right)$ having spectral gap at -1 . Assume that the corresponding Neumann quadratic form $Q_{N}$ is $G$-invariant. Then we have the following cases: 
(i) If $[v(g), U]=0$ for all $g \in G$, then $Q_{U}$ is $G$-invariant. Its closure is also $G$-invariant and the self-adjoint extension of the minimal Laplacian representing the closed quadratic form will also be $G$-invariant.

(ii) Consider the decomposition of the boundary Hilbert space $\mathcal{L}^{2}(\partial \Omega) \cong W \oplus$ $W^{\perp}$, where $W$ is the eigenspace associated with the eigenvalue -1 of $U$ and denote by $P_{W}$ the orthogonal projection onto $W$. If $Q_{U}$ is $G$-invariant and $P_{W}: \mathcal{H}^{1 / 2}(\partial \Omega) \rightarrow \mathcal{H}^{1 / 2}(\partial \Omega)$ continuous, then $[v(g), U]=0$ for all $g \in G$.

Proof. (i) Assume first that $[v(g), U]=0, g \in G$. To show that $Q_{U}$ is $G$-invariant we have to analyze first the block structure of $U$ and $v(g)$ with respect to the decomposition $\mathcal{L}^{2}(\partial \Omega) \cong W \oplus W^{\perp}$. Since

$$
U \cong\left(\begin{array}{cc}
-\mathbb{I} & 0 \\
0 & U_{0}
\end{array}\right) \quad \text { and } \quad v(g) \cong\left(\begin{array}{ll}
v_{1}(g) & v_{2}(g) \\
v_{3}(g) & v_{4}(g)
\end{array}\right)
$$

the commutation relations imply that $v_{2}(g)\left(\mathbb{I}+U_{0}\right)=0$ and $\left[v_{4}(g), U_{0}\right]=0$. But since $U$ has spectral gap at -1 , then $\mathbb{I}+U_{0}$ is invertible on $W^{\perp}$ and we must have $v_{2}(g)=0$. The unitarity of $v(g)$ implies $v_{3}(g)=0$ and $v(g)$ has block diagonal structure.

By assumption $Q_{N}$ is $G$-invariant, so it is enough to show that the boundary quadratic form

$$
B(\Phi):=\left\langle\gamma(\Phi), A_{U} \gamma(\Phi)\right\rangle_{\partial \Omega}, \quad \Phi \in \mathcal{D}_{U}
$$

is also $G$-invariant, i.e., $V(g) \mathcal{D}_{U} \subset \mathcal{D}_{U}$ and $B(V(g) \Phi)=B(\Phi), \Phi \in \mathcal{D}_{U}$. To show the first inclusion, note that for any $\Phi \in \mathcal{D}_{U}$ we have

$$
P_{W} \gamma(V(g) \Phi)=P_{W} v(g) \gamma(\Phi)=v(g) P_{W} \gamma(\Phi)=0,
$$

where we have used that $V$ is traceable along $\partial \Omega, v(g)$ has diagonal block structure and $P_{W} \cong\left(\begin{array}{cc}\mathbb{I} & 0 \\ 0 & 0\end{array}\right)$.

Finally, for any $g \in G$ and $\Phi \in \mathcal{D}_{U}$ we check

$$
\begin{aligned}
B(V(g) \Phi) & =\left\langle v_{4}(g) \gamma(\Phi), A_{U} v_{4}(g) \gamma(\Phi)\right\rangle_{W^{\perp}}=\left\langle v_{4}(g) \gamma(\Phi), v_{4}(g) A_{U} \gamma(\Phi)\right\rangle_{W^{\perp}} \\
& =\left\langle\gamma(\Phi), A_{U} \gamma(\Phi)\right\rangle_{W^{\perp}}=B(\Phi) .
\end{aligned}
$$

Note that all scalar products refer to $W^{\perp}$ and that for the last equation we used $v_{4}(g) \in\left\{U_{0}\right\}^{\prime}$ iff $v_{4}(g) \in\left\{A_{U}\right\}^{\prime}$ and, again, all commutants are taken with respect to $W^{\perp}$ (cf., Sect. 5). Since $Q_{U}$ is $G$-invariant and closable and the representation $V$ unitary it is straightforward to show that its closure is also G-invariant (see, e.g., Theorem VI.1.17 in [21]). By Theorem 4.2 it follows that the self-adjoint extension of the minimal Laplacian representing the closed quadratic form will also be $G$-invariant.

(ii) By assumption we have that

$$
P_{W}: \mathcal{H}^{1 / 2}(\partial \Omega) \rightarrow \mathcal{H}^{1 / 2}(\partial \Omega)
$$

is continuous in the fractional Sobolev norm and, therefore, $\gamma\left(\mathcal{D}_{U}\right)$ is dense in $W^{\perp}$. Since $Q_{U}$ is $G$-invariant we have $V(g) \mathcal{D}_{U} \subset \mathcal{D}_{U}$, hence for any $\Phi \in \mathcal{D}_{U}$

$$
0=P_{W} \gamma(V(g) \Phi)=P_{W} v(g) \gamma(\Phi)=P_{W} v(g) P_{W}^{\perp} \gamma(\Phi) .
$$


From the density of $\gamma\left(\mathcal{D}_{U}\right)$ in $W^{\perp}$ we conclude that $v(g) \cong\left(\begin{array}{cc}v_{1}(g) & 0 \\ 0 & v_{4}(g)\end{array}\right)$ and we only need to show $\left[v_{4}(g), U_{0}\right]=0$ on $W^{\perp}$. But this follows from the $G$-invariance of the boundary quadratic form $B$ and, again, the density of $\gamma\left(\mathcal{D}_{U}\right)$ in $W^{\perp}$

We can now consider the following immediate consequences.

Corollary 6.11. Let $U \in \mathcal{U}\left(\mathcal{L}^{2}(\partial \Omega)\right)$ be admissible and such that $\mathbb{I}+U$ is invertible. Then $Q_{U}$ on $\mathcal{D}_{U}$ is $G$-invariant iff $[v(g), U]=0$ for all $g \in G$.

Proof. We only need to show that the $G$-invariance implies that the unitaries on the boundary commute. Note that by assumption $-1 \notin \sigma(U)$ and, with the notation in the preceding theorem, we have that $W=\{0\}$. Therefore $\mathcal{D}_{U}=\mathcal{H}^{1}(\Omega)$ and the corresponding trace give $\gamma\left(D_{U}\right)=\mathcal{H}^{1 / 2}(\partial \Omega)$ which is dense in $\mathcal{L}^{2}(\partial \Omega)$. The rest of the reasoning is literally as in the proof of part (ii) of Theorem 6.10.

We conclude giving a characterization of $G$-invariance of the quadratic form $Q_{U}$ that uses the point spectrum of the unitary $U$. Recall that $\lambda$ is in the point spectrum of an operator $T$ if $(\lambda-T)$ is not injective, i.e., $\lambda$ is an eigenvalue of $T$. We denote the set of all eigenvalues by $\sigma_{p}(T) \subset \sigma(T)$.

Lemma 6.12. Consider a unitary $U \in \mathcal{U}\left(\mathcal{L}^{2}(\partial \Omega)\right)$ with spectral gap at -1 . Assume that $\mathcal{H}^{1 / 2}(\partial \Omega)$ is invariant for $U$ and that its restriction

$$
U_{+}:=U \mid \mathcal{H}^{1 / 2}(\partial \Omega)
$$

is continuous with respect to the Sobolev $1 / 2$-norm. If $U_{+}$has only point spectrum, i.e., $\sigma\left(U_{+}\right)=\sigma_{p}\left(U_{+}\right)$, then $U$ is admissible, i.e., the partial Cayley transform $A_{U}$ leaves the fractional Sobolev space $\mathcal{H}^{1 / 2}(\partial \Omega)$ invariant and is continuous with respect to the Sobolev 1/2-norm. The orthogonal projection $P_{W}$ leaves also the Sobolev space $\mathcal{H}^{1 / 2}(\partial \Omega)$ invariant and is continuous with respect to the 1/2-norm.

Proof. Note first that

$$
\sigma\left(U_{+}\right) \subset \sigma_{p}\left(U_{+}\right) \subset \sigma_{p}(U) \subset \sigma(U)
$$

and, therefore, if $U$ has spectral gap at -1 , then $U_{+}$has also spectral gap at -1 . Then by Cauchy-Riesz functional calculus (cf., [14, Chapter VII]) we have that

$$
A_{U}=\frac{1}{2 \pi i} \quad c_{1} \quad i \frac{\lambda-1}{\lambda+1}(\lambda-U)^{-1} \mathrm{~d} \lambda \quad \text { and } \quad P_{W}=\frac{1}{2 \pi i} \quad(\lambda-U)^{-1} \mathrm{~d} \lambda,
$$

where $c_{1}$ and $c_{2}$ are closed, simple and positively oriented curves. The curve $c_{1}$ encloses $\sigma(T) \backslash\{-1\}$ and $c_{2}$ encloses only $\{-1\}$. Note that the the gap condition is essential here. It is clear that both $A_{U}$ and $P_{W}$ are bounded operators in $\mathcal{H}^{1 / 2}(\partial \Omega)$ as required. 
Corollary 6.13. Consider a unitary $U \in \mathcal{U}\left(\mathcal{L}^{2}(\partial \Omega)\right)$ with spectral gap at -1 . Assume that $\mathcal{H}^{1 / 2}(\partial \Omega)$ is invariant for $U$, that its restriction $U_{+}:=$ $U \mid \mathcal{H}^{1 / 2}(\partial \Omega)$ is continuous with respect to the Sobolev $1 / 2$-norm and that $\sigma\left(U_{+}\right)=\sigma_{p}\left(U_{+}\right)$. Then $Q_{U}$ on $\mathcal{D}_{U}$ is $G$-invariant iff $[v(g), U]=0$ for all $g \in G$.

Proof. By the preceding lemma we have that $U$ is admissible and that $P_{W}$ is continuous in the $1 / 2$-norm. The statement follows then directly from Theorem 6.10 .

In the final section, we will present examples with unitaries which satisfy the conditions mentioned in the statements above.

\subsection{Groups Acting by Isometries}

We will discuss now the important instance when the unitary representation is determined by an action of the group $G$ on $\Omega$ by isometries. Thus, assume that the group $G$ acts smoothly by isometries on the Riemannian manifold $(\Omega, \partial \Omega, \eta)$. Any $g \in G$ specifies a diffeomorphism $g: \Omega \rightarrow \Omega$ that we will denote with the same symbol for simplicity of notation. Moreover, we have that

$$
g^{*} \eta=\eta,
$$

where $g^{*}$ stands for the pull-back by the diffeomorphism $g$. These diffeomorphisms restrict to isometric diffeomorphisms on the Riemannian manifold at the boundary $(\partial \Omega, \partial \eta)$ (see, e.g., [1, Lemma 8.2.4]),

$$
\left(\left.g\right|_{\partial \Omega}\right)^{*} \partial \eta=\partial \eta
$$

These isometric actions of the group $G$ induce unitary representations of the group on $\Omega$ and $\partial \Omega$. In fact, consider the following representations:

$$
\begin{aligned}
& V: G \rightarrow \mathcal{U}\left(\mathcal{L}^{2}(\Omega)\right), \quad V(g) \Phi=\left(g^{-1}\right)^{*} \Phi \quad \Phi \in \mathcal{L}^{2}(\Omega) . \\
& v: G \rightarrow \mathcal{U}\left(\mathcal{L}^{2}(\partial \Omega)\right), \quad v(g) \varphi=\left(\left.g\right|_{\partial \Omega} ^{-1}\right)^{*} \varphi \quad \varphi \in \mathcal{L}^{2}(\partial \Omega) .
\end{aligned}
$$

Then a simple computation shows that,

$$
\left\langle V\left(g^{-1}\right) \Phi, V\left(g^{-1}\right) \Psi\right\rangle=\langle\Phi, \Psi\rangle,
$$

where we have used the change of variables formula and the fact that isometric diffeomorphisms preserve the Riemannian volume, i.e., $g^{*} \mathrm{~d} \mu_{\eta}=\mathrm{d} \mu_{\eta}$. The result for the boundary is proved similarly. The induced actions are related with the trace map as

$$
\gamma(V(g) \Phi)=v(g) \gamma(\Phi) \quad g \in G, \Phi \in \mathcal{H}^{1}(\Omega),
$$

and therefore the unitary representation $V$ is traceable along the boundary of $\Omega$ with trace $v$.

Moreover, we have that the quadratic form $Q_{N}$ is $G$-invariant.

Proposition 6.14. Let $G$ be a Lie group that acts by isometric diffeomorphisms on the Riemannian manifold $(\Omega, \partial \Omega, \eta)$ and let $V: G \rightarrow \mathcal{U}\left(\mathcal{L}^{2}(\Omega)\right)$ be the associated unitary representation. Then, Neumann's quadratic form $Q_{N}(\Phi)=$ $\langle\mathrm{d} \Phi, \mathrm{d} \Phi\rangle$ with domain $\mathcal{H}^{1}(\Omega)$ is $G$-invariant. 
Proof. First notice that the pull-back of a diffeomorphism commutes with the action of the exterior differential. Then we have that

$$
\mathrm{d}\left(V\left(g^{-1}\right) \Phi\right)=\mathrm{d}\left(g^{*} \Phi\right)=g^{*} \mathrm{~d} \Phi .
$$

Hence

$$
\begin{aligned}
\left\langle\mathrm{d}\left(V\left(g^{-1}\right) \Phi\right), \mathrm{d}\left(V\left(g^{-1}\right) \Psi\right)\right\rangle & ={ }_{\Omega} \eta^{-1}\left(g^{*} \mathrm{~d} \Phi, g^{*} \mathrm{~d} \Psi\right) \mathrm{d} \mu_{\eta} \\
& ={ }_{\Omega} g^{*}\left(\eta^{-1}(\mathrm{~d} \Phi, \mathrm{d} \Psi)\right) g^{*} \mathrm{~d} \mu_{\eta} \\
& =\underset{g \Omega}{{ }_{g}} \eta^{-1}(\mathrm{~d} \Phi, \mathrm{d} \Psi) \mathrm{d} \mu_{\eta} \\
& =\langle\mathrm{d} \Phi, \mathrm{d} \Psi\rangle,
\end{aligned}
$$

where in the second inequality we have used that $g: \Omega \rightarrow \Omega$ is an isometry and therefore

$$
\eta^{-1}\left(g^{*} \mathrm{~d} \Phi, g^{*} \mathrm{~d} \Psi\right)=g^{*} \eta^{-1}\left(g^{*} \mathrm{~d} \Phi, g^{*} \mathrm{~d} \Psi\right)=g^{*}\left(\eta^{-1}(\mathrm{~d} \Phi, \mathrm{d} \Psi)\right) .
$$

The Eq. (6.2) guaranty also that $V(g) \mathcal{H}^{1}(\Omega)=\mathcal{H}^{1}(\Omega)$ since $V(g)$ is a unitary operator in $\mathcal{L}^{2}(\Omega)$ and the norm $\sqrt{\|\mathrm{d} \cdot\|_{\Lambda^{1}}^{2}+\|\cdot\|^{2}}$ is equivalent to the Sobolev norm of order 1.

Before making explicit the previous structures in concrete examples we summarize the the main ideas in this section as follows: given a group acting by isometric diffeomorphisms on a Riemannian manifold, then any operator at the boundary, that satisfies the conditions in Definitions 6.2 and 6.4, and that verifies the commutation relations of Theorem 6.10 (i) describes a $G$-invariant quadratic form. The closure of this quadratic form characterizes uniquely a $G$-invariant self-adjoint extension of the Laplace-Beltrami operator (cf. Theorems 2.7 and 4.2 ).

\section{Examples}

In this section, we introduce two particular examples of $G$-invariant quadratic forms. In the first example, we are considering a situation where the symmetry group is a finite, discrete group. In the second one, we consider $G$ to be a compact Lie group.

Example 7.1. Let $\Omega$ be the cylinder $[-1,1] \times[-1,1] / \sim$, where $\sim$ is the equivalence relation $(x, 1) \sim(x,-1)$. The boundary $\partial \Omega$ is the disjoint union of the two circles $\Gamma_{1}=\{\{-1\} \times[-1,1] / \sim\}$ and $\Gamma_{2}=\{\{1\} \times[-1,1] / \sim\}$, (see Fig. 1). Let $\eta$ be the euclidean metric on $\Omega$. Now let $G=\mathbb{Z}_{2}=\{\mathrm{e}, \mathrm{f}\}$ be the discrete, abelian group of two elements and consider the following action in $\Omega$ :

$$
\begin{aligned}
& e:(x, y) \rightarrow(x, y), \\
& f:(x, y) \rightarrow(-x, y) .
\end{aligned}
$$




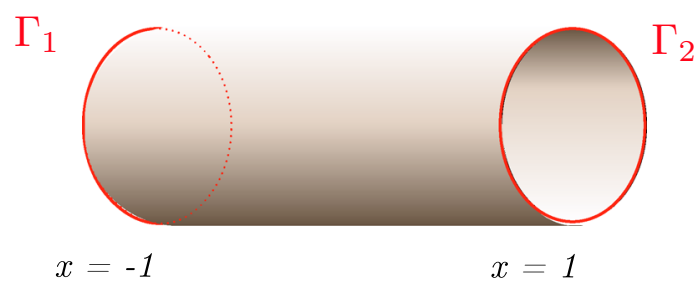

Figure 1. Representation of the cylinder in Example 7.1. $\Gamma_{1}$ and $\Gamma_{2}$ are the disconnected components of the boundary

The induced action at the boundary is

$$
\begin{aligned}
& e:( \pm 1, y) \rightarrow( \pm 1, y), \\
& f:( \pm 1, y) \rightarrow(\mp 1, y) .
\end{aligned}
$$

Clearly $G$ transforms $\Omega$ onto itself and preserves the boundary. Moreover, it is easy to check that $f^{*} \eta=\eta$. Since the boundary $\partial \Omega$ consists of two disjoints manifolds $\Gamma_{1}$ and $\Gamma_{2}$, the Hilbert space of the boundary is $\mathcal{L}^{2}(\partial \Omega)=$ $\mathcal{L}^{2}\left(\Gamma_{1}\right) \oplus \mathcal{L}^{2}\left(\Gamma_{2}\right)$. Any $\Phi \in \mathcal{L}^{2}(\partial \Omega)$ can be written as

$$
\Phi=\left(\begin{array}{l}
\Phi_{1}(y) \\
\Phi_{2}(y)
\end{array}\right)
$$

with $\Phi_{i} \in \mathcal{L}^{2}\left(\Gamma_{i}\right)$. The only nontrivial action on $\mathcal{L}^{2}(\partial \Omega)$ is given by

$$
v(f)\left(\begin{array}{l}
\Phi_{1}(y) \\
\Phi_{2}(y)
\end{array}\right)=\left(\begin{array}{ll}
0 & \mathbb{I} \\
\mathbb{I} & 0
\end{array}\right)\left(\begin{array}{l}
\Phi_{1}(y) \\
\Phi_{2}(y)
\end{array}\right) .
$$

The set of unitary operators that describe the closable quadratic forms as defined in the previous section is given by suitable unitary operators

$$
U=\left(\begin{array}{ll}
U_{11} & U_{12} \\
U_{21} & U_{22}
\end{array}\right)
$$

with $U_{i j}=\mathcal{L}^{2}\left(\Gamma_{j}\right) \rightarrow \mathcal{L}^{2}\left(\Gamma_{i}\right)$. According to Theorem 6.10 (i) the unitary operators commuting with $v(f)$ will lead to $G$-invariant quadratic forms. Imposing

$$
\left(\begin{array}{ll}
0 & \mathbb{I} \\
\mathbb{I} & 0
\end{array}\right)\left(\begin{array}{ll}
U_{11} & U_{12} \\
U_{21} & U_{22}
\end{array}\right)=\left(\begin{array}{ll}
U_{11} & U_{12} \\
U_{21} & U_{22}
\end{array}\right)\left(\begin{array}{ll}
0 & \mathbb{I} \\
\mathbb{I} & 0
\end{array}\right)
$$

we get the conditions

$$
\begin{aligned}
& U_{21}-U_{12}=0, \\
& U_{22}-U_{11}=0 .
\end{aligned}
$$

Obviously there is a wide class of unitary operators, i.e., boundary conditions, that will be compatible with the symmetry group $G$. We will consider next two particular classes of boundary conditions. First, consider the following unitary operators

$$
U=\begin{array}{cc}
e^{\mathrm{i} \beta_{1}} \mathbb{I}_{1} & 0 \\
0 & e^{\mathrm{i} \beta_{2}} \mathbb{I}_{2}
\end{array}
$$


where $\beta_{i} \in \mathcal{C}^{\infty}\left(S^{1},[-\pi+\delta, \pi-\delta] \cup\{\pi\}\right)$ for some $\delta>0$. It is showed in [19, Sections 3 and 5] that this class of unitary operators have spectral gap at -1 and are admissible (see also Sect. 6.2). Moreover, this choice of unitary matrices corresponds to select Robin boundary conditions of the form:

$$
\left.\gamma\left(-\frac{\mathrm{d} \Phi}{\mathrm{d} x}\right)\right|_{\Gamma_{1}}=-\left.\tan \left(\beta_{1} / 2\right) \gamma(\Phi)\right|_{\Gamma_{1}} ;\left.\gamma\left(\frac{\mathrm{d} \Phi}{\mathrm{d} x}\right)\right|_{\Gamma_{2}}=-\left.\tan \left(\beta_{2} / 2\right) \gamma(\Phi)\right|_{\Gamma_{2}} .
$$

The $G$-invariance condition above imposes $\beta_{1}=\beta_{2}$. Notice that when $\beta_{1} \neq$ $\beta_{2}$ we can obtain meaningful self-adjoint extensions of the Laplace-Beltrami operator that, however, will not be $G$-invariant.

We can also consider unitary operators of the form

$$
U=\begin{array}{cc}
0 & e^{\mathrm{i} \alpha} \\
e^{-\mathrm{i} \alpha} & 0
\end{array},
$$

where $\alpha \in \mathcal{C}^{\infty}\left(S^{1},[0,2 \pi]\right)$. Again, it is proved in [19] that this class of unitary operators have spectral gap at -1 and are admissible. In this case, the unitary matrix corresponds to select so-called quasi-periodic boundary conditions, cf. [4], i.e.,

$$
\begin{gathered}
\left.\gamma(\Phi)\right|_{\Gamma_{1}}=\left.e^{i \alpha} \gamma(\Phi)\right|_{\Gamma_{2}}, \\
\left.\gamma\left(-\frac{\mathrm{d} \Phi}{\mathrm{d} x}\right)\right|_{\Gamma_{1}}=\left.e^{i \alpha} \gamma\left(\frac{\mathrm{d} \Phi}{\mathrm{d} x}\right)\right|_{\Gamma_{2}} .
\end{gathered}
$$

The $G$-invariance condition imposes $e^{i \alpha}=e^{-i \alpha}$ and therefore among all the quasi-periodic conditions only the periodic ones, $\alpha \equiv 0$, are compatible with the $G$-invariance.

Example 7.2. Let $\Omega$ be the unit, upper hemisphere centered at the origin. Its boundary $\partial \Omega$ is the unit circle on the $z=0$ plane. Let $\eta$ be the induced Riemannian metric from the euclidean metric in $\mathbb{R}^{3}$. Consider the compact Lie group $G=S O(2)$ that acts by rotation around the $z$-axis. If we use polar coordinates on the horizontal plane, then the boundary $\partial \Omega$ is isomorphic to the interval $[0,2 \pi]$ with the two endpoints identified. We denote by $\theta$ the coordinate parameterizing the boundary and the boundary Hilbert space is $\mathcal{L}^{2}\left(S^{1}\right)$.

Let $\varphi \in \mathcal{H}^{1 / 2}(\partial \Omega)$ and consider the action on the boundary by a group element $g_{\alpha} \in G, \alpha \in[0,2 \pi]$, given by

$$
v\left(g_{\alpha}^{-1}\right) \varphi(\theta)=\varphi(\theta+\alpha) .
$$

To analyze what are the possible unitary operators that lead to $G$-invariant quadratic forms it is convenient to use the Fourier series expansions of the elements in $\mathcal{L}^{2}(\partial \Omega)$. Let $\varphi \in \mathcal{L}^{2}(\partial \Omega)$, then

$$
\varphi(\theta)=\sum_{n \in \mathbb{Z}} \hat{\varphi}_{n} e^{\mathrm{i} n \theta},
$$


where the coefficients of the expansion are given by

$$
\hat{\varphi}_{n}=\frac{1}{2 \pi}{ }_{0}^{2 \pi} \varphi(\theta) e^{-\mathrm{i} n \theta} \mathrm{d} \theta .
$$

We can therefore consider the induced action of the group $G$ as a unitary operator on $\ell_{2}$, the Hilbert space of square summable sequences. In fact we have that:

$$
\begin{aligned}
\left(\widehat{\left.v\left(g_{\alpha}^{-1}\right) \varphi\right)_{n}}\right. & =\frac{1}{2 \pi}{ }_{0}^{2 \pi} \varphi(\theta+\alpha) e^{-\mathrm{i} n \theta} \mathrm{d} \theta \\
& =\sum_{m \in \mathbb{Z}} \hat{\varphi}_{m} e^{\mathrm{i} m \alpha}{ }_{0}^{2 \pi} \frac{e^{\mathrm{i}(m-n) \theta}}{2 \pi} \mathrm{d} \theta=e^{\mathrm{i} n \alpha} \hat{\varphi}_{n} .
\end{aligned}
$$

This shows that the induced action of the group $G$ is a unitary operator in $\mathcal{U}\left(\ell_{2}\right)$ that acts diagonally in the Fourier series expansion. More concretely, we can represent it as $\widehat{v\left(g_{\alpha}^{-1}\right)_{n m}}=e^{\mathrm{i} n \alpha} \delta_{n m}$. From all the possible unitary operators acting on the Hilbert space of the boundary, only those whose representation in $\ell_{2}$ commutes with the above operator will lead to $G$-invariant quadratic forms (cf., Theorem 6.10 (i)). Since $\overline{v\left(g_{\alpha}^{-1}\right)}$ acts diagonally on $\ell_{2}$ it is clear that only operators of the form $\hat{U}_{n m}=e^{\mathrm{i} \beta_{n}} \delta_{n m},\left\{\beta_{n}\right\}_{n} \subset \mathbb{R}$, will lead to $G$-invariant quadratic forms.

As a particular case we can consider that all the parameters are equal, i.e., $\beta_{n}=\beta, n \in \mathbb{Z}$. In this case it is clear that $(\widehat{U \varphi})_{n}=e^{\mathrm{i} \beta} \varphi_{n}$, which gives the following admissible unitary with spectral gap at -1 :

$$
U \varphi=e^{\mathrm{i} \beta} \varphi .
$$

This shows that the unique Robin boundary conditions compatible with the $S O(2)$-invariance are those that are defined with a constant parameter along the boundary, i.e.,

$$
\gamma\left(\frac{\mathrm{d} \Phi}{\mathrm{d} \vec{n}}\right)=-\tan (\beta / 2) \gamma(\Phi), \quad \beta \in[0,2 \pi],
$$

where $\vec{n}$ stands for normal vector field pointing outwards to the boundary.

\section{References}

[1] Abraham, R., Marsden, J., Ratiu, T.: Manifolds, Tensor Analysis and Applications. Springer, Berlin (1988)

[2] Adams, R.A., Fournier, J.J.F.: Sobolev Spaces. Pure and Applied Mathematics, 2nd edn. Academic Press, Oxford (2003)

[3] Akhiezer, N.I., Glazman, I.M.: Theory of Linear Operators in Hilbert Spaces, vol. II. Dover Publications, New York (1961)

[4] Asorey, M., Esteve, J.G., Pacheco, A.F.: Planar rotor: the $\theta$-vacuum structure, and some approximate methods in Quantum Mechanics. Phys. Rev. D 27, 18521868 (1983)

[5] Asorey, M., García-Álvarez, D., Muñoz-Castañeda, J.M.: Casimir effect and global theory of boundary conditions. J. Phys. A 39, 6127-6136 (2006) 
[6] Bargmann, V.: On unitary ray representations of continuous groups. Ann. Math. 59, 1-46 (1954)

[7] Bekker, M.B.: A class of nondensely defined Hermitian contractions. Adv. Dyn. Syst. Appl. 2, 141-165 (2007)

[8] Bekker, M.B., Tsekanovskii, E.: On periodic matrix-valued Weyl-Titchmarsh functions. J. Math. Anal. Appl. 294, 666-686 (2004)

[9] Berezanskii, J.M.: Expansions in Eigenfunctions of Self-adjoint Operators. Translations of Monographs. American Mathematical Society, Providence (1968)

[10] Borchers, H.J., Yngvason, J.: Positivity of Wightman functionals and the existence of local nets. Commun. Math. Phys. 127, 607-615 (1990)

[11] Cariñena, J.F., Santander, M.: On the projective unitary representations of connected Lie groups. J. Math. Phys. 16, 1416-1420 (1975)

[12] Cariñena, J.F., Santander, M.: Projective covering group versus representation groups. J. Math. Phys. 21, 440-443 (1980)

[13] Davies, E.B.: Spectral Theory and Differential Operators. Cambridge University Press, London (1995)

[14] Dunford, N., Schwartz, J.T.: Linear Operators Part I: General Theory. Wiley, New York (1976)

[15] Dunford, N., Schwartz, J.T.: Linear Operators Part II: Spectral Theory. Self Adjoint Operators in Hilbert Space. Wiley, New York (1963)

[16] Exner, P., Fraas, F.: On the dense point and absolutely continuous spectrum for Hamiltonians with concentric $\delta$ shells. Lett. Math. Phys. 82, 25-37 (2007)

[17] Dittrich, J., Exner, P., Seba, P.: Dirac Hamiltonian with Coulomb potential and spherically symmetric shell contact interaction. J. Math. Phys. 33, 2207-2214 (1992)

[18] Gitman, D.M., Tyutin, I.V., Voronov, B.L.: Self-Adjoint Extensions in Quantum Mechanics. Birkäuser, New York (2012)

[19] Ibort, A., Lledó, F., Pérez-Pardo, J.M.: Self-adjoint extensions of the LaplaceBeltrami operator and unitaries at the boundary. Preprint 2013. arXiv:1308.0527 (to appear in J. Funct. Anal.)

[20] Ibort, A., Pérez-Pardo, J.M.: Numerical solutions of the spectral problem for arbitrary self-adjoint extensions of the one-dimensional Schrödinger equation. SIAM J. Numer. Anal. 51, 1254-1279 (2013)

[21] Kato, T.: Perturbation Theory for Linear Operators. Classics in Mathematics. Springer, Berlin (1995)

[22] Koshmanenko, V.: Singular Quadratic Forms in Perturbation Theory. Kluwer Academic Publishers, Dordrecht (1999)

[23] Lions, J.L., Magenes, E.: Non-Homogeneous Boundary Value Problems and Applications. Grundlehren der mathematischen Wissenschaften, vol. I. Springer, Berlin (1972)

[24] Mackey, G.W.: The Theory of Unitary Group Representations. The University of Chicago Press, Chicago (1976)

[25] Moretti, V.: Spectral Theory and Quantum Mechanics. Springer, Milan (2013)

[26] Nussbaum, A.E.: Reduction theory for unbounded closed operators in Hilbert space. Duke Math. J. 31, 33-44 (1964) 
[27] Pedersen, G.K.: Analysis Now. Springer, New York (1989)

[28] Posilicano, A.: Self-adjoint extensions of restrictions. Oper. Matrices 2, 483-506 (2008)

[29] Reed, M., Simon, B.: Methods of Modern Mathematical Physics, vol. I. Academic Press, New York (1980)

[30] Reed, M., Simon, B.: Methods of Modern Mathematical Physics, vol. II. Academic Press, New York (1975)

[31] Simon, B.: Quantum dynamics: from automorphisms to hamiltonians. In: Lieb, E.H., Simon, B., Wightman, A.S. (eds.) Studies in Mathematical Physics. Essays in Honor of Valentine Bargman, Princeton University Press, Princeton (1976)

[32] Thaller, B.: The Dirac Equation. Springer, Berlin (1992)

[33] von Neumann, J.: Zur Algebra der Funktionaloperationen und der Theorie der normalen Operatoren. Math. Ann. 102, 307-427 (1929)

[34] von Neumann, J.: Allgemeine Eigenwerttheorie hermitescher Funktionaloperatoren. Math. Ann. 102, 49-131 (1930)

[35] Schmüdgen, K.: Unbounded Self-Adjoint Operators on Hilbert Space. Springer, Dordrecht (2012)

[36] Shams, N., Stanhope, E., Webb, D.L.: One cannot hear orbifold isotropy type. Arch. Math. 87, 375-384 (2006)

[37] van der Waerden, B.L.: Die gruppentheoretische Methode in der Quantenmechanik. Julius Springer, Berlin (1932)

[38] Weyl, H.: Gruppentheorie und Quantenmechanik. S. Hirzel, Leipzig (1928)

[39] Wigner, E.P.: On unitary representations of the inhomogeneous Lorentz group. Ann. Math. 40, 149-204 (1939)

[40] Wigner, E.P.: Gruppentheorie und ihre Anwendung auf die Quantenmechanik der Atomspektren. F. Vieweg und Sohn, Braunschweig (1931)

[41] Woronowicz, S.L.: Unbounded elements affiliated with $\mathrm{C}^{*}$-algebras and noncompact quantum groups. Commun. Math. Phys. 136, 399-432 (1991)

[42] Woronowicz, S.L., Napiórkowski, K.: Operator theory in the C*-algebra framework. Rep. Math. Phys. 31, 353-371 (1992)

Alberto Ibort, Fernando Lledó and Juan Manuel Pérez-Pardo

Department of Mathematics

Universidad Carlos III de Madrid

Avda. de la Universidad 30

28911 Leganés (Madrid), Spain

e-mail: albertoi@math.uc3m.es;

flledo@math.uc3m.es

Alberto Ibort, Fernando Lledó and Juan Manuel Pérez-Pardo Instituto de Ciencias Matemáticas (CSIC-UAM-UC3M-UCM) c./ Nicolás Cabrera 13-15

Campus de Cantoblanco, UAM

28049 Madrid, Spain

Juan Manuel Pérez-Pardo

INFN-Sezione di Napoli

Via Cintia Edificio 6

80126 Naples, Italy

e-mail: juanma@na.infn.it 